[Frontiers in Bioscience, Elite, 5, 558-573, January 1, 2013]

\title{
T-cell activation induces selective changes of cellular lipidome
}

Tapio Lonnberg ${ }^{1}$, Laxman Yetukuri², Tuulikki Seppanen-Laakso ${ }^{2}$, Riitta Lahesmaa ${ }^{1}$, Matej Oresic ${ }^{2}$

${ }^{I}$ Turku Centre for Biotechnology, University of Turku and Abo Akademi University, Turku, Finland, ${ }^{2}$ VTT Technical Research Centre of Finland, Espoo, Finland

\section{TABLE OF CONTENTS}

1. Abstract

2. Introduction

3. Material and methods

3.1. $\mathrm{CD}^{+}$cell isolation

3.2. Cell stimulation and culture

3.3. Harvesting

3.4. Lipidomic analysis

4. Results

3.5. Clustering analysis

4.1. Lipid composition of naïve T helper precursor cells

4.2. Alterations of lipid concentrations in response to $T$-cell activation

4.3. Changes in PC and PE fatty acid change lengths and degree of saturation during development of activated T-cell phenotypes

5. Discussion

4.4. Transcriptional regulation of lipid metabolism

6. Acknowledgements

7. References

\section{ABSTRACT}

Activation of naïve $\mathrm{T}$ helper cells by presentation of cognate antigen initiates a complex intracellular signaling process leading to development of functionally active effector cell population. The switch from quiescent naïve state to activated state involves a profound change of cellular metabolism, required for completion of multiple rounds of proliferation. Using ultra performance liquid chromatography mass spectrometry, we analyzed how this change is reflected on the cellular lipid composition in human umbilical cord blood T-cells. We found that considerable concentration changes take place during the first 72 hours after T-cell receptor activation, correlating with first rounds of activation-induced cell division. Most importantly, composition of phosphatidylcholines and phosphatidylethanolamines exhibited consistent trend towards shorter and more saturated molecular species. Together with related transcriptomics data, the results clearly suggested induction of de novo fatty acid synthesis and accumulation of endogenously synthesized fatty acids into the cellular membranes, leading to partial remodeling of the cellular lipidome in the newly developed effector cell population.

\section{INTRODUCTION}

$\mathrm{T}$ helper cells constitute the topmost regulatory layer of the adaptive immune system, controlling the activity of all downstream immune cell types and thus coordinating all acquired immune responses. The activity of $\mathrm{T}$ helper cells themselves is determined by $\mathrm{T}$-cell receptor-mediated activation by cognate peptide-MHC complexes and subsequent cytokine-influenced differentiation of functionally distinct effector subsets, namely Th1, Th2, and Th17 cells(1-3). These processes are prerequisites for the specificity and memory that are the hallmark features of acquired immune responses.

Since the fundamental function of T-cell activation is to recognize pathogenic non-self peptides in an environment dominated by innocuous and self molecules, extremely tight and specific regulation is required. Importantly, contrary to the functions of many other cell surface receptors, T-cell activation does not follow typical mass action kinetics where rate of the reaction is determined by concentrations of participating molecules. Instead, remarkably complex network of intracellular 
signaling mechanisms has evolved that controls the initiation, duration, and strength of activation(4-6).

Essentially, the current model holds that binding of antigen peptide-MHC complex to TCR $\alpha / \beta$ subunits leads to phosphorylation of cytoplasmic ITAM domains and is followed by autophosphorylation of ZAP70. Phosphorylation of ZAP70, in turn, leads to activation of key scaffold proteins LAT and SLP76, enabling signal diversification into multiple pathways, most importantly Ras, PKC $\theta$, and intracellular $\mathrm{Ca}^{2+}$-mediated signaling. Experimental evidence from recent high-throughput studies suggests that ultimately $\mathrm{T}$-cell receptor activation has profound effects on cellular function, as illustrated by phosphorylation of more than 400 proteins already at one hour after stimulation(7), and differential activation of over 6000 genes within 48 hours after activation(8).

Maintenance of an appropriate lipid composition in the cellular membranes is required to ensure membrane fluidity, topology of attached proteins, activity of membrane-bound enzymes, degree of exposure of surface proteins, lateral mobility of receptors and activation of specific signaling pathways. Given such an importance of membrane lipid composition, cells have developed robust mechanisms to maintain the membrane lipid homeostasis. To understand T-cell lipid metabolism, it is thus important to measure its end products, the cellular lipids, at the molecular level. The so-called lipidomics approach, covering a global profile of structurally and functionally diverse lipids, may not only elucidate the lipid molecular composition of T-cells, but also deliver clues about the mechanisms behind the control of cellular lipid homeostasis in the process of T-cell activation(9).

To which extent the signals leading to T-cell activation are reflected on cellular lipid composition has so far not been addressed in a system-wide fashion. Until recent advances in mass spectrometry-based approaches(911), this limitation has been arguably attributed more to lack of efficient tools than to lack of interest, as the specific roles of certain lipids in the proximal stages of TCR signaling are well established. Particularly, activation of both Ras and PKC $\theta$ pathways are dependent on production of diacylglycerol (DG) from plasma membrane phosphatidylinositol 4,5 bisphosphate $\left(\mathrm{PI}(4,5) \mathrm{P}_{2}\right)$ by phospholipase $\mathrm{C} \gamma$ (PLC $\gamma$ ) in response to TCR engagement. The other product of this reaction, inositol $(1,4,5)$ triphospate $\left(\mathrm{IP}_{3}\right)$, in turn, mediates release of cytoplasmic $\mathrm{Ca}^{2+}$ and initiation of $\mathrm{Ca}^{2+}$-mediated signaling(12). Moreover, targeted approaches have been recently exploited e.g. in the context of lipid-ordered plasma membrane domains(13), and have provided strong evidence in support of regulatory roles for specific molecular lipid environments. Therefore the question arises whether also the more downstream steps of T-cell activation and consequent development of effector cell subsets are regulated by lipids or mediated by lipid intermediates.

Here we present global analysis of cellular lipid profiles in human primary umbilical cord blood T-cells both in unstimulated state and during a 72-hour time course following T-cell activation. In addition, cumulative effects of IL-4, the key Th2-promoting cytokine, were measured. The results indicated significant longitudinal changes in concentrations of several glycerophospholipids. Importantly, a systematic increase in the concentrations of saturated, short-chain phosphatidylcholines and phosphatidylethanolamines was measured, with a corresponding decrease in longer, polyunsaturated species.

To our knowledge our data currently represents
the most complete catalogue of cellular
glycerophospholipids, sphingolipids, and triglycerides from
primary human T-cells, and suggests considerable plasticity
in the global lipid composition of activated T-cells.

\section{MATERIAL AND METHODS}

\section{1. $\mathrm{CD}^{+}$cell isolation}

Umbilical cord blood samples were obtained from healthy neonate donors from Turku University Central Hospital. Mononuclear cells were isolated using Ficoll gradient centrifugation (Amersham). $\mathrm{CD}^{+}$cells were further purified using positive selection with antiCD4-coated magnetic Dynal beads (Invitrogen). The typical purity of such samples has been determined to be in the order of $98-99 \%(63)$

\subsection{Cell stimulation and culture}

$\mathrm{CD}^{+}$cells were cultured in Yssel's medium (Iscove modified Dulbecco medium [IMDM; Invitrogen] supplemented with Yssel medium concentrate, penicillin, streptomycin, and $1 \% \mathrm{AB}$-serum) at a density of $2-3 * 10^{6}$ cells $/ \mathrm{ml}$ on 24 -well plates. Cells were activated using platebound anti-CD3 $(0.5 \mu \mathrm{g} / \mathrm{well})$ and soluble anti-CD28 $(0.5 \mu \mathrm{g} / \mathrm{ml})$, (both antibodies from Beckman Coulter). Th2 polarization was induced with the addition of IL-4 (10 ng/ml, R\&D Systems).

\subsection{Harvesting}

Cells were harvested in unstimulated state and at $0.5,1,2,4,6,12,24,48$, and 72 hours after activation. Harvesting and metabolic quenching was performed as described by de Koning et al.(64), with some modifications. In brief, culture medium was removed; cells were resuspended to $-20^{\circ} \mathrm{C} 50 \%$ methanol, and pelleted by centrifugation $(770 \mathrm{~g} 20 \mathrm{~min})$ at $-20^{\circ} \mathrm{C}$. The supernatant was discarded and cells were stored in liquid nitrogen.

\subsection{Lipidomic analysis}

Aliquots of cultured T-cells containing $c a .2$ million cells were spiked with a standard mixture consisting of 10 lipid compounds $(0.2 \mu \mathrm{g} / \mathrm{sample})$ and mixed with $100 \mu$ l of chloroform/methanol (2:1) by vortexing for $2 \mathrm{~min}$. After $1 \mathrm{~h}$ standing the tubes were centrifuged at $10000 \mathrm{rpm}$ for $3 \mathrm{~min}$ and the lower organic phase was separated into a vial insert and mixed with a standard mixture containing 3 labelled lipid compounds $(0.1 \mu \mathrm{g} / \mathrm{sample})$.

Lipidomics analysis was performed as described previously(65). Briefly, the lipid extracts were run on a Waters Q-Tof Premier mass spectrometer (Waters) 
combined with an Acquity Ultra Performance Liquid Chromatography ${ }^{\mathrm{TM}}$ (Waters) by using a solvent system including 1) water with $1 \% 1 \mathrm{M} \mathrm{NH}_{4} \mathrm{Ac}$ and $0.1 \% \mathrm{HCOOH}$ and 2) LC/MS grade acetonitrile/isopropanol (5:2) with $1 \%$ $1 \mathrm{M} \mathrm{NH} \mathrm{NH}_{4} \mathrm{Ac}, 0.1 \% \mathrm{HCOOH}$. The gradient from $65 \% \mathrm{~A} /$ $35 \% \mathrm{~B}$ to $100 \% \mathrm{~B}$ lasted for $6 \mathrm{~min}$ and the total run time including a $5 \mathrm{~min}$ re-equilibration step was $18 \mathrm{~min}$. An Acquity UPLC ${ }^{\mathrm{TM}}$ BEH C18 $1 \times 50 \mathrm{~mm}$ column with 1.7 $\mu \mathrm{m}$ particles was used at $50^{\circ} \mathrm{C}$ and at a flow rate of 0.200 $\mathrm{ml} / \mathrm{min}$. The lipid profiling was carried out using ESI+ mode and the data was collected at mass range of $\mathrm{m} / \mathrm{z} 300$ 1200 .

Data was processed using MZmine 2 software(66). Lipid identification was performed using inhouse spectral library as described previously (67). The relevant lipid species in the library were identified as follows: In positive ion mode the molecular species of PC form either protonated $[\mathrm{M}+\mathrm{H}]^{+}$or sodiated $[\mathrm{M}+\mathrm{Na}]^{+}$adduct ions as well as a dominant peak at $\mathrm{m} / \mathrm{z} 184$, representing the choline head group. Additionally, the sodiated PC adducts form characteristic peaks at $\mathrm{m} / \mathrm{z}[\mathrm{M}+\mathrm{Na}-59]^{+}$, $[\mathrm{M}+\mathrm{Na}-205]^{+}$and $[\mathrm{M}+\mathrm{Na}-183]^{+}$. Ether lipid identification is usually based on the accurate $\mathrm{m} / \mathrm{z}$ values (usually protonated or sodiated adduct) as well as MS/MS level diagnostic fragment ion at $\mathrm{m} / \mathrm{z} 184$ in positive ion mode. Negative electrospray ionisation was used when details concerning the fatty acid composition was required. As PEs are zwitterionic, both positive and negative electrospray could be used to characterize their fragmentation. PE molecular species form protonated species $[\mathrm{M}+\mathrm{H}]^{+}$that yield a major fragment ion at $\mathrm{m} / \mathrm{z}[\mathrm{M}+\mathrm{H}-141]^{+}$(due to loss of the head group) in ESI-MS/MS positive mode. Plasmalogen (etherlinked) ethanolamines are detected based on two fragmental ions characteristic of sn-1 and sn2 positions. ESI-MS of TG species yield ammonium adduct ions that are fragmented in MS/MS to produce diacylglycerol $\left([D G]^{+}\right)$like fragments, which are informative in identifying TG species. ESI-MS is not well suited for the analysis of free cholesterol. Cholesteryl esters, however, form ammonium adducts in positive ion mode as well as a fragment ion at $\mathrm{m} / \mathrm{z} 369$ upon collision-induced fragmentation. In positive mode, ESI-MS analysis of sphingolipids, such as sphingomyelin (SM), form a characteristic protonated phosphocholine peak at m/z 184 . PC and SM species are distinguished on the basis of their characteristic $\mathrm{m} / \mathrm{z}$ value (PC species occur at even at $\mathrm{m} / \mathrm{z}$ and SM species at odd $\mathrm{m} / \mathrm{z}$ ). SM species yield either $[\mathrm{M}+\mathrm{H}]^{+}$or $[\mathrm{M}+\mathrm{Na}]^{+}$ions in positive ionization mode and $[\mathrm{M}-\mathrm{CH} 3]^{-}$and $[\mathrm{M}+45]^{-}$ions with negative ionisation. Ceramide species form unstable protonated molecular species which undergo dehydration to form $\left[\mathrm{M}+\mathrm{H}-\mathrm{H}_{2} \mathrm{O}\right]^{+}$ peaks in ESI positive ion mode. The molecular ions observed in negative ionisation conditions are very informative in identifying the fatty acyl and long chain base substituents of ceramide. Ceramide species yield $[\mathrm{M}-\mathrm{H}]^{-}$ and $[\mathrm{M}-\mathrm{H}-30]^{-}$(due to loss of $\mathrm{HCHO}$ group) ions in negative ionisation conditions. While positive mode analysis of long chain bases such as sphingosine (d18:1), sphinganine (d18:0) and 4-D-hydroxysphinganine (t18:0) undergo dehydration to form fragments at $\mathrm{m} / \mathrm{z} 282 / 264$, $284 / 266$ and 300/282, negative ion mode analysis of sphingosine and phytosphingosine moieties are characterised based on the fragments at $\mathrm{m} / \mathrm{z}$ 237/263 and 225/255/267 respectively.

The normalization of lipidomics data was performed as follows: all monoacyl lipids except cholesterol esters, such as monoacylglycerols and monoacylglycerophospholipids were normalized with PC (17:0/0:0), all diacyl lipids except ethanolamine phospholipids were normalized with PC(17:0/17:0), ceramides with $\operatorname{Cer}(\mathrm{d} 18: 1 / 17: 0)$, the diacyl ethanolamine phospholipids were normalized with $\operatorname{PE}(17: 0 / 17: 0)$, and the TG and cholesterol esters with TG(17:0/17:0/17:0).

\subsection{Clustering analysis}

Clustering analysis was performed using $\mathrm{R}$ (version 2.10.1) employing Ward distance metrics.

\section{RESULTS}

\subsection{Lipid composition of naïve $T$ helper precursor cells}

The lipid composition of unstimulated human $\mathrm{T}$ helper cells was analyzed from samples of CD4-purified human umbilical cord blood cells using ultra performance liquid chromatography mass spectrometry (UPLC-MS). The analyses were carried out using four biological replicate samples, each representing a pool of cells from multiple individuals.

As a result, in total 41 distinct lipid species could be reproducibly identified. Most of these represented phosphatidylcholines (PC) and phosphatidylethanolamines (PE), which are the most abundant lipid classes both in terms of identified species, and absolute concentration, as illustrated in Figure 1. The other main classes of lipids analyzed included sphingomyelins (SM), triacylglycerols (TG), and diacylglycerols. The identification strategy applied resulted in the assignment of lipid sum formula for each of the measured species, which consisted of the headgroup class, total number of fatty acyl side chain carbon atoms, and the number of side chain double bonds: e.g. PC(32:0) indicating a phosphatidylcholine with carbon number of 32 and no double bonds.

\subsection{Alterations of lipid concentrations in response to $T$ - cell activation}

Activation through T-cell receptor has profound influence on transcriptional and translational activity. To investigate how these effects are reflected on concentrations of cellular lipids, UPLC-MS-based lipidomic analyses were performed for ex vivo cultured cord blood $\mathrm{CD}^{+}$T-cells during a 72-hour time series consisting of nine time points following activation with plate-bound anti-CD3 and soluble co-stimulatory antiCD28 antibodies. The activation was performed in presence or absence of IL-4, the key Th2-promoting cytokine, to analyze whether cytokine-induced Th subset differentiation had additional cumulative effects during this time course.

Altogether 67 lipids were successfully quantified from all samples. To gain an overview on the regulation of these lipids, hierarchical clustering analysis was performed 

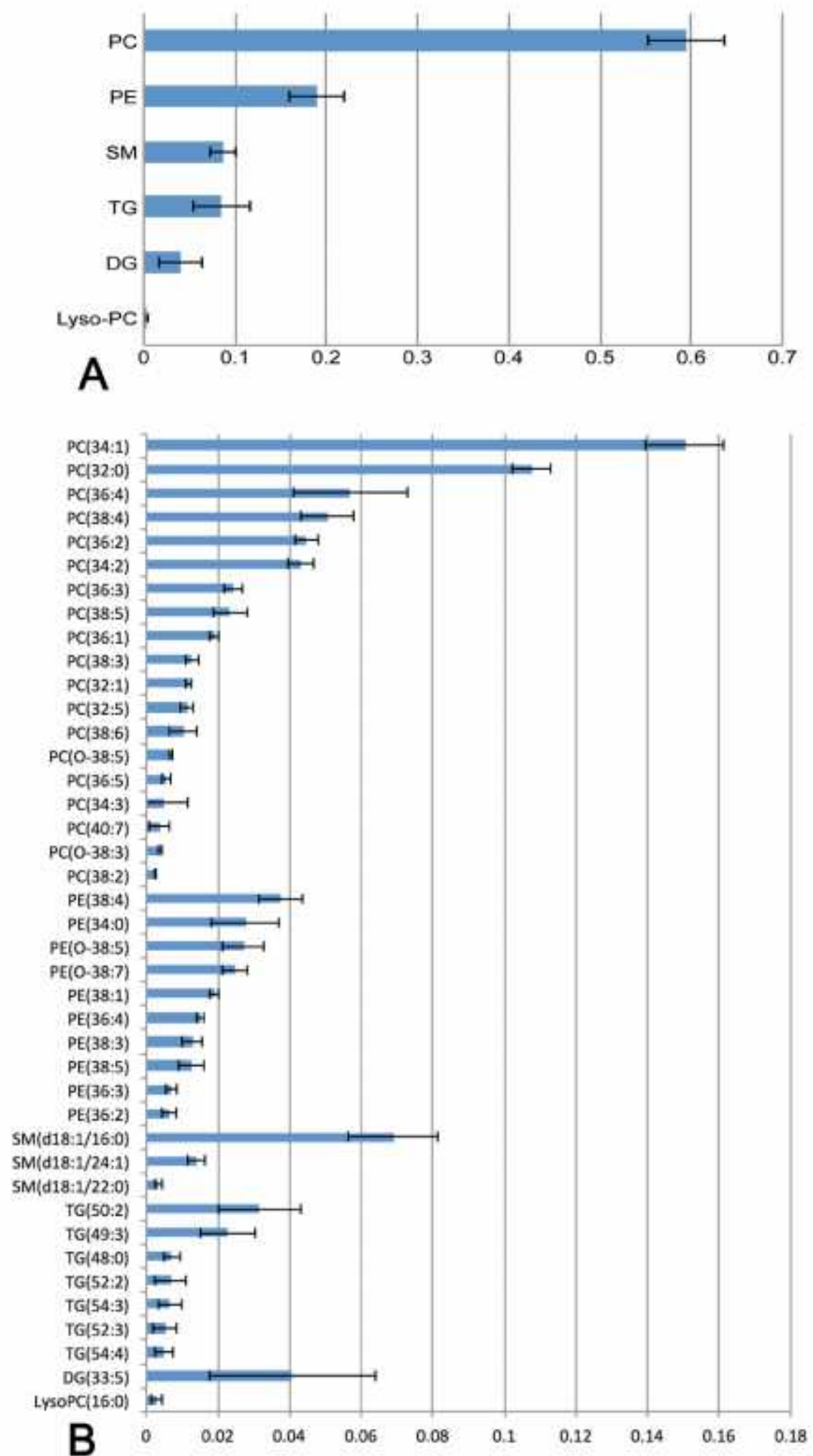

Figure 1. Relative lipid abundance profiles in unstimulated naïve $\mathrm{CD} 4^{+}$T-cells. Lipid class concentrations (1A) were calculated as the sum of individual lipid species concentrations and are represented in relation to the total amount of all identified lipids. Individual lipid species concentrations were normalized in a similar manner to the total amount of all the identified species (1B). The values are averages of four biological replicates. Error bars indicate standard deviation values. 


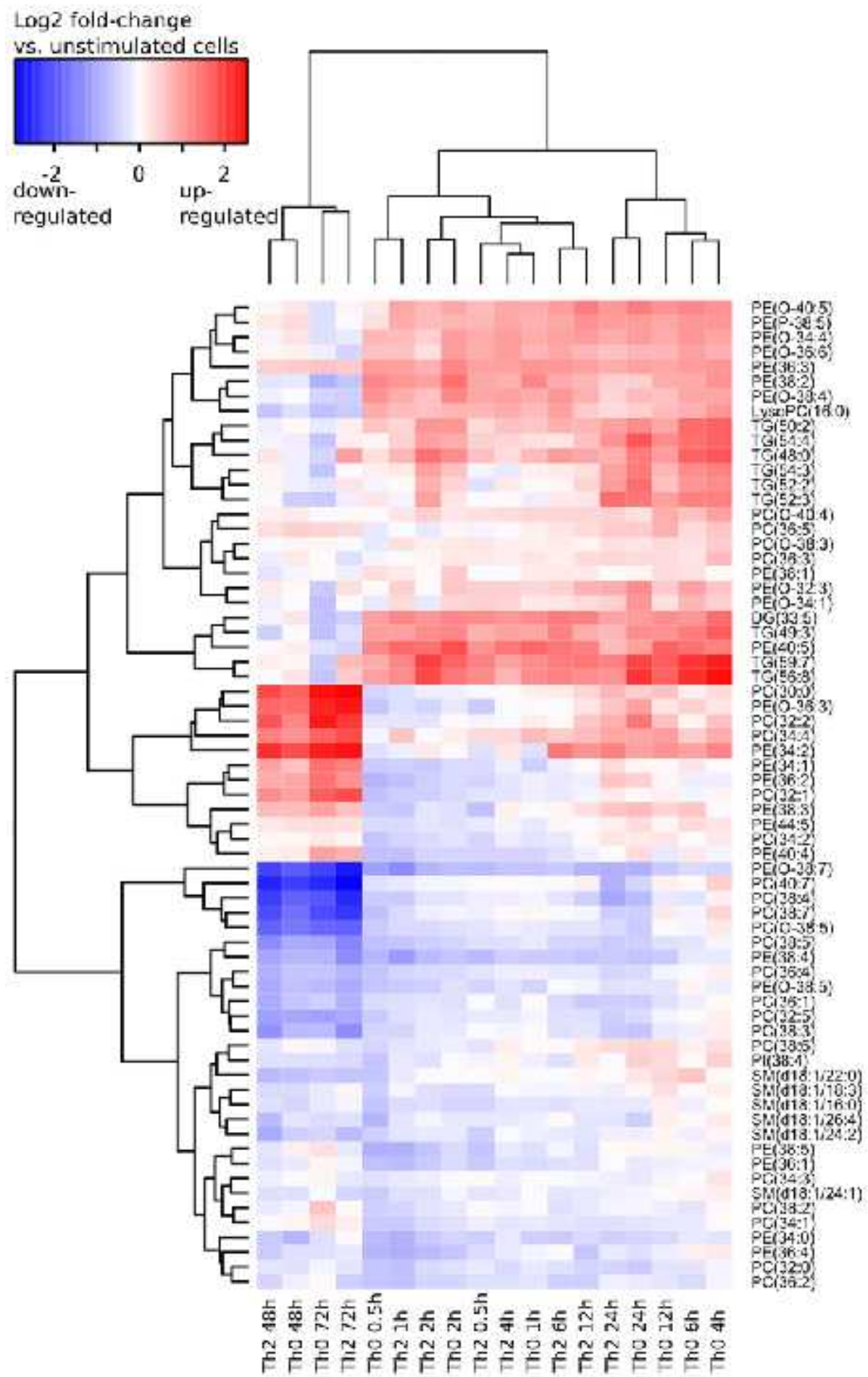

Figure 2. Clustering analysis of lipid time-series concentration profiles in response to T-cell activation. Concentration values were normalized to the measured concentration in the unstimulated samples, and are expressed as log2-based fold changes in comparison to the unstimulated sample, blue indicating down-regulation and red indicating up-regulation. Th0 stands for cells activated in absence of cytokine stimulation, Th2 stands for cells activated in presence of IL- 4 .

both in sample-direction as well as in lipid species time kinetics-axis. The results indicated that the later time points (48 and 72 hours) exhibited the most distinct changes, while earlier time points clustered more closely together (Figure 2). The potential cumulative effect of IL-4 stimulation appeared to be all but negligible, and in general 

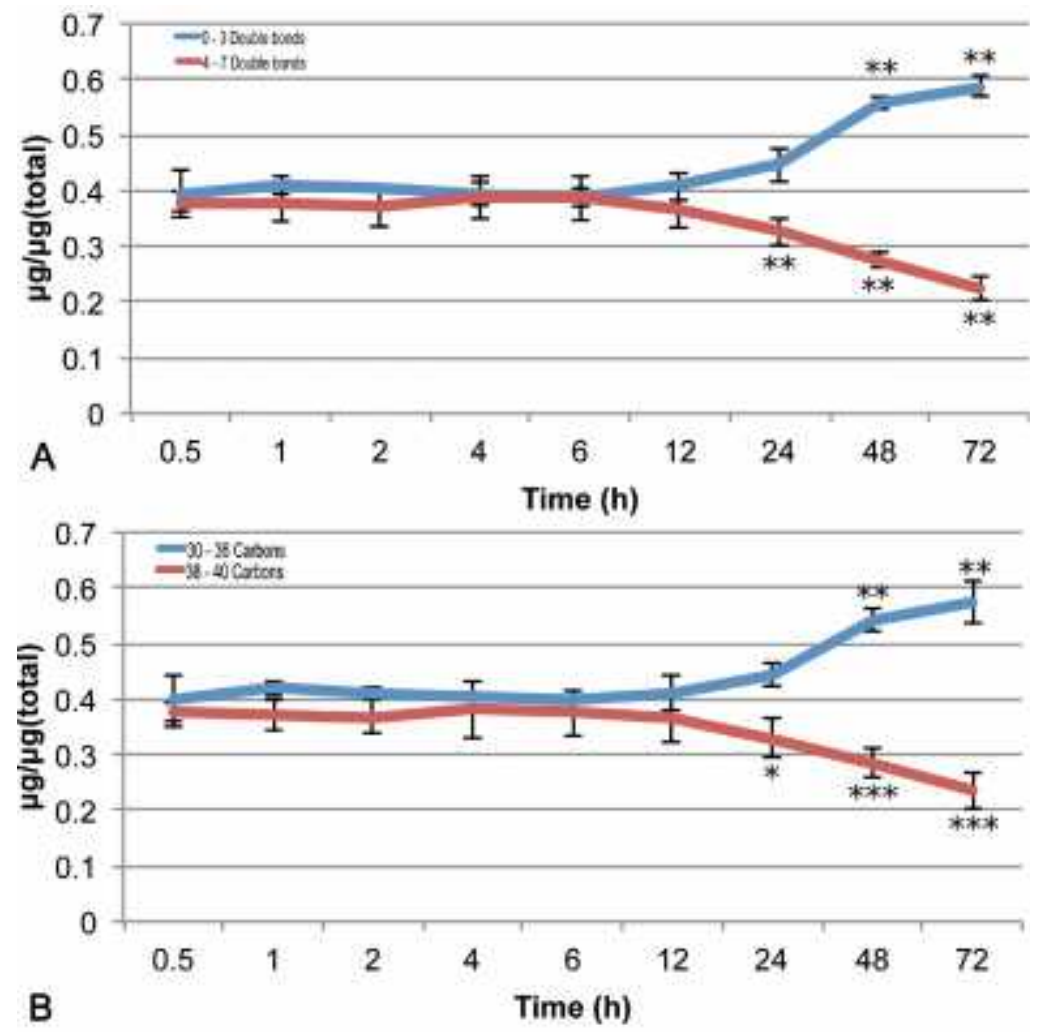

Figure 3. Relative amounts of phosphatidylcholine and phosphatidylethanolamine lipids in activated T-cells categorized by fatty acid residue double bond number $(3 \mathrm{~A})$ or carbon number (3B). Concentrations are presented in relation to cumulative concentration of all identified lipid species, while time refers to hours after CD3/CD28-mediated activation. Values are averages from cells cultured in presence or absence of IL-4 from two independent experiments. Error bars represent standard deviation values. P-values were calculated using Student's paired t-test comparing each time point to corresponding measurements from $0.5 \mathrm{~h}$ time point and marked as significance levels: $* \mathrm{p}<0.05 ; * * \mathrm{p}<0.01 ; * * * \mathrm{p}<0.001$.

could not be reliably distinguished from biological variation, thus making time point the determining factor in sample-direction clustering.

As a result of clustering in kinetics-direction, the identified lipids exhibited at least four distinct types of regulation: lipids that are at first upregulated, but decrease in later time points; lipids that increase towards the end of the time series; lipids that decrease towards the end of time series, and ones that remain relatively constant throughout the time series. The time-course concentration profiles of all the species analyzed are provided in Tables 1 and 2.

\subsection{Changes in $P C$ and $P E$ fatty acid chain lengths and degree of saturation during development of activated $\mathrm{T}$ - cell phenotypes}

The clustering analysis indicated that the most pronounced concentration changes were associated with lipid species of PC and PE classes. A closer inspection of the results suggested that differentially regulated clusters consisted of lipid species with distinct fatty acid compositions. Interestingly, lipids that were downregulated at 48 and 72 hours seemed to be enriched with polyunsaturated species, while upregulated lipid species contained on the average notably smaller amount of double bonds. To further validate these quantitative changes in PC and PE fatty acids and their potential implications on cell membrane composition, the experiment was reproduced using cells isolated from cord blood samples from different individuals. The lipid species were quantified using the established workflow, resulting in identification of 34 PC and $\mathrm{PE}$ species that were in common with the original dataset.

To investigate the correlation of relative abundance with lipid saturation and side chain length, all 34 identified PC and PE lipids were grouped on the basis of side chain carbon number ( 36 , or $\geq 38$ ), and side chain double bond number ( 3 , or $\geq 4$ ), and their cumulative relative concentration calculated. Time series analysis of these lipid species was in line with the finding that in activated cells shorter and less saturated fatty acids are in general favored over polyunsaturated species, and that these changes are significant in terms of total lipid concentrations, resulting in increasing remodeling of the cellular lipidome during the first 72 hours following activation (Figure 3). The specificity of the concentration changes was also obvious at the level of individual lipid species (Figure 4). In the comparison of the earliest and latest time points ( 0.5 and 72 hours), the most significantly 
Human T helper cell lipidomics

Table 1. Lipid concentrations in Th0 cells compared to unstimulated cells ( $\log 2)$

\begin{tabular}{|c|c|c|c|c|c|c|c|c|c|}
\hline Name & $0.5 \mathrm{~h}$ & 1h & $2 \mathrm{~h}$ & 4h & 6h & $12 \mathrm{~h}$ & $24 \mathrm{~h}$ & $48 \mathrm{~h}$ & $72 \mathrm{~h}$ \\
\hline DG(33:5) & 0.91 & 1.16 & 1.21 & 1.47 & 1.02 & 1.16 & 0.98 & 0.23 & -0.71 \\
\hline LysoPC(16:0) & 0.81 & 0.65 & 0.73 & 1.02 & 0.79 & 0.66 & 0.44 & -0.31 & -0.69 \\
\hline PC(30:0) & -0.45 & 0.13 & -0.13 & 0.34 & 0.11 & 0.63 & 0.47 & 1.55 & 2.40 \\
\hline $\mathrm{PC}(32: 0)$ & -0.62 & -0.21 & -0.35 & -0.08 & -0.30 & -0.30 & -0.59 & -0.35 & -0.10 \\
\hline $\mathrm{PC}(32: 1)$ & -0.72 & -0.13 & -0.42 & 0.09 & -0.08 & 0.15 & 0.04 & 0.91 & 1.61 \\
\hline $\mathrm{PC}(32: 2)$ & -0.25 & 0.11 & 0.04 & 0.61 & 0.06 & 0.63 & 1.36 & 1.22 & 2.32 \\
\hline $\mathrm{PC}(32: 5)$ & -0.60 & -0.12 & -0.30 & 0.13 & 0.01 & -0.07 & -0.42 & -0.99 & -1.04 \\
\hline $\mathrm{PC}(34: 1)$ & -0.55 & -0.20 & -0.36 & 0.03 & -0.22 & -0.21 & -0.30 & 0.05 & 0.35 \\
\hline $\mathrm{PC}(34: 2)$ & -0.63 & -0.25 & -0.27 & 0.18 & 0.03 & 0.25 & 0.18 & 0.08 & 0.18 \\
\hline $\mathrm{PC}(34: 3)$ & -0.36 & -0.01 & -0.06 & 0.23 & -0.03 & -0.08 & -0.24 & 0.00 & 0.07 \\
\hline $\mathrm{PC}(34: 4)$ & -0.11 & 0.68 & 0.12 & 0.83 & 0.51 & 1.01 & 0.89 & 1.08 & 1.54 \\
\hline $\mathrm{PC}(36: 1)$ & -0.40 & 0.01 & -0.31 & 0.00 & -0.14 & -0.32 & -0.50 & -0.67 & -0.50 \\
\hline $\mathrm{PC}(36: 2)$ & -0.62 & -0.26 & -0.48 & -0.12 & -0.40 & -0.27 & -0.24 & -0.14 & 0.00 \\
\hline $\operatorname{PC}(36: 3)$ & -0.09 & 0.26 & 0.18 & 0.64 & 0.22 & 0.34 & 0.34 & 0.16 & 0.04 \\
\hline $\mathrm{PC}(36: 4)$ & -0.62 & -0.16 & -0.38 & 0.15 & -0.06 & -0.08 & -0.40 & -0.65 & -0.63 \\
\hline $\mathrm{PC}(36: 5)$ & -0.24 & 0.15 & 0.07 & 0.55 & 0.33 & 0.76 & 0.52 & 0.46 & 0.40 \\
\hline $\mathrm{PC}(38: 2)$ & -0.49 & -0.02 & -0.24 & 0.04 & -0.30 & -0.13 & -0.11 & -0.09 & 0.54 \\
\hline $\mathrm{PC}(38: 3)$ & -0.49 & -0.04 & -0.19 & 0.13 & -0.16 & -0.24 & -0.62 & -0.76 & -0.74 \\
\hline $\mathrm{PC}(\mathrm{O}-38: 3)$ & -0.22 & 0.15 & 0.16 & 0.53 & 0.28 & 0.32 & 0.26 & 0.10 & 0.07 \\
\hline $\mathrm{PC}(38: 4)$ & -0.55 & -0.10 & -0.30 & 0.16 & -0.27 & -0.23 & -0.77 & -1.82 & -1.96 \\
\hline $\operatorname{PC}(38: 5)$ & -0.72 & -0.19 & -0.43 & -0.10 & -0.30 & -0.34 & -0.52 & -0.92 & -0.99 \\
\hline $\mathrm{PC}(\mathrm{O}-38: 5)$ & -0.58 & -0.19 & -0.36 & 0.17 & -0.09 & -0.05 & -0.60 & -1.55 & -1.76 \\
\hline $\mathrm{PC}(38: 6)$ & -0.45 & 0.05 & -0.17 & 0.27 & 0.11 & 0.34 & 0.42 & 0.08 & -0.07 \\
\hline $\mathrm{PC}(38: 7)$ & -0.68 & 0.06 & -0.08 & 0.42 & -0.07 & 0.19 & -0.50 & -1.62 & -2.06 \\
\hline $\mathrm{PC}(\mathrm{O}-40: 4)$ & 0.01 & 0.41 & 0.25 & 0.94 & 0.60 & 0.81 & 0.31 & 0.16 & -0.01 \\
\hline $\mathrm{PC}(40: 7)$ & -0.31 & -0.04 & -0.07 & 0.45 & 0.03 & 0.14 & -0.46 & -2.21 & -2.50 \\
\hline $\mathrm{PC}(50: 3)$ & 0.77 & 1.47 & 1.37 & 1.77 & 1.36 & 1.63 & 1.65 & 0.18 & -0.58 \\
\hline $\mathrm{PE}(\mathrm{O}-32: 3)$ & 0.01 & 0.41 & 0.57 & 0.55 & 0.91 & 0.51 & 1.12 & 0.03 & -0.58 \\
\hline $\mathrm{PE}(34: 0)$ & -0.77 & -0.49 & -0.43 & -0.30 & -0.21 & -0.37 & -0.47 & -0.83 & -0.31 \\
\hline $\operatorname{PE}(34: 1)$ & -0.56 & -0.51 & -0.40 & -0.03 & 0.22 & 0.04 & 0.10 & 0.62 & 1.41 \\
\hline $\mathrm{PE}(\mathrm{O}-34: 1)$ & -0.21 & 0.19 & 0.39 & 0.45 & 0.73 & 0.28 & 1.02 & -0.01 & -0.70 \\
\hline $\mathrm{PE}(34: 2)$ & -0.35 & -0.32 & -0.06 & 1.21 & 0.90 & 1.07 & 0.93 & 1.77 & 2.26 \\
\hline $\mathrm{PE}(\mathrm{O}-34: 4)$ & 0.72 & 0.80 & 0.96 & 0.90 & 1.01 & 0.83 & 0.96 & 0.22 & -0.34 \\
\hline $\operatorname{PE}(36: 1)$ & -0.70 & -0.41 & -0.38 & -0.10 & -0.07 & -0.17 & -0.23 & -0.23 & 0.07 \\
\hline $\operatorname{PE}(36: 2)$ & -0.80 & -0.17 & -0.38 & -0.16 & -0.15 & 0.08 & 0.44 & 0.90 & 1.41 \\
\hline $\mathrm{PE}(36: 3)$ & 1.00 & 1.02 & 1.21 & 1.06 & 0.97 & 0.87 & 0.91 & 0.51 & 0.56 \\
\hline $\mathrm{PE}(\mathrm{O}-36: 3)$ & -0.65 & 0.05 & -0.19 & 0.22 & 0.47 & 0.26 & 0.94 & 1.47 & 2.22 \\
\hline $\operatorname{PE}(36: 4)$ & -0.84 & -0.11 & -0.64 & 0.18 & 0.14 & -0.16 & -0.31 & -0.35 & -0.36 \\
\hline PE(O-36:6) & 0.64 & 0.71 & 0.96 & 0.77 & 0.95 & 0.69 & 0.71 & 0.14 & -0.18 \\
\hline $\mathrm{PE}(38: 1)$ & 0.28 & 0.20 & 0.50 & 0.05 & 0.30 & 0.35 & 0.06 & -0.08 & 0.02 \\
\hline $\operatorname{PE}(38: 2)$ & 1.21 & 1.18 & 1.41 & 1.07 & 0.84 & 0.80 & 0.48 & -0.19 & -0.89 \\
\hline $\operatorname{PE}(38: 3)$ & -0.59 & -0.02 & -0.33 & -0.02 & 0.53 & 0.42 & 0.67 & 0.69 & 0.92 \\
\hline $\mathrm{PE}(38: 4)$ & -0.85 & -0.59 & -0.62 & -0.19 & -0.24 & -0.57 & -0.64 & -0.83 & -0.82 \\
\hline $\mathrm{PE}(\mathrm{O}-38: 4)$ & 1.11 & 0.85 & 1.18 & 0.88 & 0.87 & 0.59 & 0.64 & 0.00 & -0.48 \\
\hline $\mathrm{PE}(38: 5)$ & -0.86 & -0.32 & -0.37 & -0.16 & 0.07 & 0.07 & 0.07 & 0.12 & 0.27 \\
\hline $\mathrm{PE}(\mathrm{O}-38: 5)$ & -0.66 & -0.45 & -0.36 & -0.17 & 0.07 & -0.25 & -0.30 & -0.68 & -0.74 \\
\hline $\mathrm{PE}(\mathrm{O}-38: 7)$ & -1.08 & -0.67 & -0.70 & -0.47 & -0.42 & -0.61 & -0.90 & -1.85 & -2.19 \\
\hline $\mathrm{PE}(40: 4)$ & -0.68 & -0.49 & -0.47 & -0.09 & 0.16 & -0.22 & 0.34 & 0.19 & 0.91 \\
\hline PE(40:5) & 1.21 & 1.53 & 1.77 & 1.27 & 1.40 & 1.57 & 0.94 & 0.11 & -0.36 \\
\hline $\mathrm{PE}(40: 5 \mathrm{e})$ & 0.17 & 0.83 & 0.89 & 1.08 & 1.17 & 1.06 & 1.30 & 0.25 & -0.36 \\
\hline $\operatorname{PE}(44: 5)$ & -0.41 & -0.24 & -0.31 & 0.22 & 0.33 & 0.07 & 0.33 & 0.31 & 0.32 \\
\hline $\mathrm{PE}(\mathrm{P}-38: 5)$ & 0.28 & 0.79 & 0.74 & 1.02 & 1.03 & 0.86 & 0.96 & 0.32 & -0.36 \\
\hline $\mathrm{PI}(38: 4)$ & -0.62 & -0.13 & -0.06 & 0.47 & 0.02 & 0.35 & 0.47 & -0.31 & -0.35 \\
\hline SM(d18:1/16:0) & -0.63 & -0.28 & -0.44 & 0.00 & -0.16 & 0.14 & -0.23 & -0.46 & -0.23 \\
\hline SM(d18:1/18:3) & -0.51 & -0.06 & -0.42 & 0.14 & 0.03 & 0.29 & 0.07 & -0.37 & -0.19 \\
\hline $\mathrm{SM}(\mathrm{d} 18: 1 / 22: 0)$ & -0.67 & -0.02 & -0.03 & 0.04 & 0.54 & 0.40 & 0.25 & -0.72 & -0.57 \\
\hline SM(d18:1/24:1) & -0.58 & 0.00 & -0.08 & 0.13 & 0.06 & 0.05 & -0.10 & -0.31 & -0.04 \\
\hline $\mathrm{SM}(\mathrm{d} 18: 1 / 24: 2)$ & -0.52 & -0.18 & -0.20 & 0.06 & -0.13 & 0.07 & -0.14 & -0.54 & -0.43 \\
\hline SM(d18:1/26:4) & -0.85 & -0.18 & -0.23 & 0.17 & 0.01 & 0.13 & -0.55 & -0.29 & -0.38 \\
\hline TG(48:0) & 0.30 & 0.64 & 1.13 & 1.65 & 1.58 & 0.96 & 1.30 & -0.13 & -0.49 \\
\hline TG(49:3) & 0.99 & 0.90 & 1.21 & 1.61 & 1.31 & 1.10 & 1.13 & -0.04 & -0.70 \\
\hline TG(50:2) & 0.56 & 0.55 & 1.03 & 1.54 & 1.43 & 0.87 & 1.16 & 0.02 & -0.22 \\
\hline TG(52:2) & -0.09 & 0.03 & 0.38 & 1.13 & 1.02 & 0.60 & 1.45 & -0.14 & -0.31 \\
\hline TG(52:3) & 0.22 & -0.15 & 0.24 & 1.24 & 1.25 & 0.94 & 1.33 & -0.50 & -0.56 \\
\hline $\mathrm{TG}(54: 3)$ & 0.14 & 0.13 & 0.63 & 1.27 & 1.16 & 0.69 & 1.31 & -0.14 & -0.66 \\
\hline TG(54:4) & 0.06 & 0.28 & 0.82 & 1.59 & 1.40 & 1.13 & 1.70 & -0.15 & -0.62 \\
\hline TG(56:8) & 0.94 & 1.16 & 1.34 & 2.42 & 2.07 & 1.59 & 2.05 & 0.23 & -0.66 \\
\hline TG(59:7) & 0.81 & 1.16 & 1.56 & 2.27 & 2.07 & 1.62 & 1.87 & 0.07 & -0.60 \\
\hline
\end{tabular}


Table 2. Lipid concentrations in Th2 cells compared to unstimulated cells ( $\log 2)$

\begin{tabular}{|c|c|c|c|c|c|c|c|c|c|}
\hline Name & $0.5 \mathrm{~h}$ & 1h & $2 \mathrm{~h}$ & $4 h$ & 6h & $12 \mathrm{~h}$ & $24 \mathrm{~h}$ & $48 \mathrm{~h}$ & $72 \mathrm{~h}$ \\
\hline DG(33:5) & 1.12 & 1.19 & 1.03 & 1.02 & 1.31 & 1.26 & 0.93 & -0.09 & -0.52 \\
\hline LysoPC(16:0) & 0.72 & 0.66 & 0.63 & 0.81 & 0.91 & 0.52 & 0.33 & -0.62 & -0.58 \\
\hline PC $(30: 0)$ & -0.05 & -0.34 & -0.13 & 0.03 & 0.16 & 0.39 & 0.73 & 1.84 & 2.52 \\
\hline $\mathrm{PC}(32: 0)$ & -0.21 & -0.70 & -0.39 & -0.42 & -0.31 & -0.44 & -0.48 & -0.27 & -0.30 \\
\hline $\mathrm{PC}(32: 1)$ & -0.28 & -0.54 & -0.51 & -0.25 & -0.24 & -0.09 & 0.20 & 1.15 & 1.75 \\
\hline $\mathrm{PC}(32: 2)$ & -0.09 & -0.29 & -0.25 & -0.01 & 0.03 & 0.58 & 0.77 & 1.74 & 2.01 \\
\hline $\mathrm{PC}(32: 5)$ & -0.20 & -0.31 & -0.24 & -0.23 & -0.32 & -0.35 & -0.49 & -1.06 & -0.95 \\
\hline $\mathrm{PC}(34: 1)$ & -0.15 & -0.56 & -0.39 & -0.31 & -0.26 & -0.35 & -0.36 & -0.03 & 0.13 \\
\hline $\mathrm{PC}(34: 2)$ & -0.44 & -0.48 & -0.43 & -0.14 & -0.23 & 0.03 & 0.22 & 0.08 & 0.07 \\
\hline $\mathrm{PC}(34: 3)$ & 0.00 & -0.19 & -0.17 & -0.13 & -0.02 & -0.09 & -0.13 & -0.18 & -0.24 \\
\hline $\mathrm{PC}(34: 4)$ & 0.33 & 0.56 & -0.06 & 0.36 & 0.52 & 0.68 & 0.86 & 1.24 & 1.80 \\
\hline $\operatorname{PC}(36: 1)$ & -0.05 & -0.31 & -0.27 & -0.36 & -0.37 & -0.55 & -0.52 & -0.93 & -0.88 \\
\hline $\mathrm{PC}(36: 2)$ & -0.31 & -0.72 & -0.46 & -0.32 & -0.53 & -0.53 & -0.17 & -0.49 & -0.50 \\
\hline $\operatorname{PC}(36: 3)$ & 0.16 & -0.01 & 0.07 & 0.12 & 0.14 & 0.35 & 0.37 & -0.17 & -0.33 \\
\hline $\mathrm{PC}(36: 4)$ & -0.25 & -0.48 & -0.38 & -0.16 & -0.22 & -0.20 & -0.48 & -0.89 & -0.80 \\
\hline $\mathrm{PC}(36: 5)$ & -0.06 & -0.01 & -0.24 & -0.06 & 0.21 & 0.33 & 0.50 & 0.27 & 0.32 \\
\hline $\mathrm{PC}(38: 2)$ & 0.01 & -0.44 & -0.25 & -0.09 & -0.32 & -0.24 & -0.11 & -0.23 & 0.02 \\
\hline $\mathrm{PC}(38: 3)$ & -0.02 & -0.46 & -0.20 & -0.09 & -0.32 & -0.30 & -0.59 & -1.23 & -1.35 \\
\hline $\mathrm{PC}(\mathrm{O}-38: 3)$ & 0.26 & 0.27 & 0.14 & 0.13 & 0.18 & 0.11 & 0.28 & -0.01 & -0.03 \\
\hline $\mathrm{PC}(38: 4)$ & -0.14 & -0.58 & -0.29 & -0.16 & -0.27 & -0.53 & -0.98 & -2.21 & -2.45 \\
\hline $\mathrm{PC}(38: 5)$ & -0.32 & -0.64 & -0.54 & -0.37 & -0.46 & -0.46 & -0.60 & -1.33 & -1.41 \\
\hline $\mathrm{PC}(\mathrm{O}-38: 5)$ & -0.27 & -0.40 & -0.42 & -0.14 & -0.29 & -0.25 & -0.39 & -1.80 & -1.82 \\
\hline $\mathrm{PC}(38: 6)$ & -0.27 & -0.28 & -0.24 & 0.15 & 0.02 & 0.16 & 0.34 & -0.30 & -0.32 \\
\hline $\operatorname{PC}(38: 7)$ & -0.16 & -0.38 & -0.16 & 0.02 & -0.02 & -0.09 & -0.53 & -2.07 & -2.27 \\
\hline $\mathrm{PC}(\mathrm{O}-40: 4)$ & 0.29 & 0.23 & 0.43 & 0.33 & 0.42 & 0.40 & 0.47 & 0.12 & 0.01 \\
\hline $\mathrm{PC}(40: 7)$ & -0.03 & -0.22 & -0.11 & 0.03 & -0.02 & 0.13 & -0.86 & -2.55 & -2.91 \\
\hline $\mathrm{PC}(50: 3)$ & 1.15 & 1.41 & 1.24 & 1.17 & 1.30 & 1.54 & 1.22 & 0.50 & 0.57 \\
\hline $\mathrm{PE}(\mathrm{O}-32: 3)$ & 0.43 & 0.45 & -0.01 & 0.46 & 0.43 & 0.74 & 0.80 & 0.16 & 0.29 \\
\hline $\operatorname{PE}(34: 0)$ & -0.49 & -0.87 & -0.59 & -0.18 & -0.60 & -0.40 & -0.53 & -0.58 & 0.00 \\
\hline $\mathrm{PE}(34: 1)$ & -0.40 & -0.52 & -0.55 & -0.14 & -0.15 & 0.04 & 0.35 & 0.95 & 1.25 \\
\hline $\mathrm{PE}(\mathrm{O}-34: 1)$ & 0.43 & 0.34 & -0.24 & 0.41 & 0.26 & 0.35 & 0.46 & -0.16 & 0.02 \\
\hline $\operatorname{PE}(34: 2)$ & -0.32 & -0.18 & 0.20 & 0.22 & 1.34 & 1.08 & 1.23 & 2.12 & 2.39 \\
\hline $\mathrm{PE}(\mathrm{O}-34: 4)$ & 0.84 & 0.66 & 0.47 & 0.98 & 0.95 & 0.98 & 0.80 & -0.10 & -0.24 \\
\hline $\operatorname{PE}(36: 1)$ & -0.57 & -0.75 & -0.60 & -0.31 & -0.36 & -0.34 & -0.02 & -0.31 & -0.08 \\
\hline $\operatorname{PE}(36: 2)$ & -0.46 & -0.70 & -0.55 & -0.26 & -0.23 & -0.13 & 0.54 & 0.80 & 1.09 \\
\hline $\operatorname{PE}(36: 3)$ & 0.94 & 0.95 & 0.76 & 0.94 & 1.06 & 0.94 & 0.80 & 0.45 & 0.48 \\
\hline $\mathrm{PE}(\mathrm{O}-36: 3)$ & -0.58 & -0.34 & -0.43 & -0.03 & -0.04 & 0.26 & 0.58 & 1.54 & 2.36 \\
\hline $\operatorname{PE}(36: 4)$ & -0.43 & -0.89 & -0.75 & -0.22 & 0.01 & -0.64 & -0.20 & -0.60 & -0.23 \\
\hline $\mathrm{PE}(\mathrm{O}-36: 6)$ & 0.83 & 0.62 & 0.31 & 0.98 & 0.87 & 0.70 & 0.61 & 0.06 & -0.44 \\
\hline $\operatorname{PE}(38: 1)$ & 0.00 & 0.17 & 0.03 & -0.01 & 0.19 & 0.21 & 0.00 & -0.28 & -0.16 \\
\hline $\mathrm{PE}(38: 2)$ & 0.88 & 1.02 & 0.95 & 0.80 & 0.80 & 0.70 & 0.44 & -0.30 & -0.66 \\
\hline $\operatorname{PE}(38: 3)$ & -0.68 & -0.62 & -0.30 & 0.12 & 0.07 & 0.30 & 0.55 & 0.67 & 0.59 \\
\hline $\operatorname{PE}(38: 4)$ & -0.75 & -1.11 & -0.77 & -0.51 & -0.57 & -0.66 & -0.56 & -0.99 & -1.29 \\
\hline $\mathrm{PE}(\mathrm{O}-38: 4)$ & 0.86 & 0.85 & 0.62 & 0.95 & 1.06 & 0.75 & 0.39 & -0.13 & -0.52 \\
\hline $\operatorname{PE}(38: 5)$ & -0.65 & -0.90 & -0.64 & -0.21 & -0.11 & -0.35 & 0.01 & -0.29 & -0.21 \\
\hline $\mathrm{PE}(\mathrm{O}-38: 5)$ & -0.44 & -0.60 & -0.52 & -0.28 & -0.06 & -0.07 & -0.36 & -0.93 & -0.95 \\
\hline $\mathrm{PE}(\mathrm{O}-38: 7)$ & -0.79 & -1.30 & -0.83 & -0.63 & -0.61 & -0.84 & -0.92 & -2.14 & -2.68 \\
\hline $\mathrm{PE}(40: 4)$ & -0.46 & -0.72 & -0.48 & -0.48 & -0.31 & -0.13 & -0.02 & 0.16 & 0.78 \\
\hline PE(40:5) & 1.09 & 1.51 & 1.39 & 1.35 & 1.35 & 1.23 & 0.65 & 0.00 & -0.48 \\
\hline $\operatorname{PE}(40: 5 \mathrm{e})$ & 0.66 & 0.89 & 0.67 & 0.87 & 1.00 & 1.29 & 1.06 & 0.10 & 0.06 \\
\hline $\mathrm{PE}(44: 5)$ & -0.21 & -0.43 & -0.28 & -0.07 & 0.06 & -0.03 & 0.15 & 0.20 & 0.27 \\
\hline $\mathrm{PE}(\mathrm{P}-38: 5)$ & 0.70 & 0.83 & 0.67 & 0.87 & 0.88 & 1.09 & 1.00 & 0.19 & -0.03 \\
\hline $\mathrm{PI}(38: 4)$ & -0.12 & -0.21 & -0.33 & 0.09 & -0.33 & 0.21 & -0.05 & -0.35 & -0.38 \\
\hline SM(d18:1/16:0) & -0.49 & -0.40 & -0.35 & -0.30 & -0.33 & -0.21 & -0.20 & -0.33 & -0.09 \\
\hline SM(d18:1/18:3) & -0.38 & -0.06 & -0.31 & -0.02 & -0.10 & -0.07 & -0.16 & -0.38 & 0.08 \\
\hline $\mathrm{SM}(\mathrm{d} 18: 1 / 22: 0)$ & 0.05 & -0.21 & 0.01 & 0.10 & -0.06 & 0.08 & 0.04 & -0.74 & -0.67 \\
\hline SM(d18:1/24:1) & -0.30 & -0.33 & -0.33 & -0.21 & -0.26 & -0.24 & -0.13 & -0.35 & -0.47 \\
\hline SM(d18:1/24:2) & -0.52 & -0.25 & -0.19 & -0.01 & -0.22 & -0.25 & -0.17 & -0.96 & -0.76 \\
\hline $\mathrm{SM}(\mathrm{d} 18: 1 / 26: 4)$ & -0.18 & -0.29 & -0.10 & -0.22 & 0.03 & -0.30 & -0.30 & -0.79 & -0.35 \\
\hline TG(48:0) & 0.61 & 0.71 & 1.40 & 0.36 & 0.97 & 0.86 & 0.79 & 0.25 & 0.91 \\
\hline TG(49:3) & 0.75 & 0.93 & 1.15 & 0.95 & 1.25 & 0.80 & 0.72 & -0.54 & -0.32 \\
\hline TG(50:2) & 0.35 & 0.40 & 1.03 & 0.59 & 0.75 & 0.72 & 0.92 & -0.13 & 0.12 \\
\hline $\mathrm{TG}(52: 2)$ & -0.32 & 0.04 & 0.75 & 0.17 & 0.09 & 0.39 & 0.97 & 0.08 & 0.26 \\
\hline TG(52:3) & 0.00 & -0.08 & 0.91 & 0.03 & 0.17 & 0.25 & 1.47 & -0.03 & -0.15 \\
\hline TG(54:3) & -0.08 & 0.18 & 0.94 & -0.19 & 0.16 & 0.29 & 0.90 & 0.10 & -0.03 \\
\hline TG(54:4) & 0.42 & 0.46 & 0.91 & 0.39 & 0.26 & 0.63 & 1.10 & -0.06 & 0.15 \\
\hline TG(56:8) & 1.12 & 1.15 & 1.85 & 0.74 & 1.23 & 1.16 & 1.09 & -0.06 & 0.48 \\
\hline TG(59:7) & 1.26 & 1.20 & 1.94 & 0.95 & 1.32 & 1.35 & 1.25 & 0.11 & 0.65 \\
\hline
\end{tabular}

upregulated PC species were relatively short-chained and saturated ( $\mathrm{PC}(30: 0), \mathrm{PC}(32: 1), \mathrm{PC}(32: 2)$, and $\mathrm{PC}(34: 2))$, while species ( $\mathrm{PC}(38: 4)$ and $\mathrm{PC}(\mathrm{O}-38: 5))$ were most clearly diminished. A similar, although less consistent tendency to shorter and less saturated lipids was also seen in in lipids of PE class. An additional regulatory pattern was observed in 


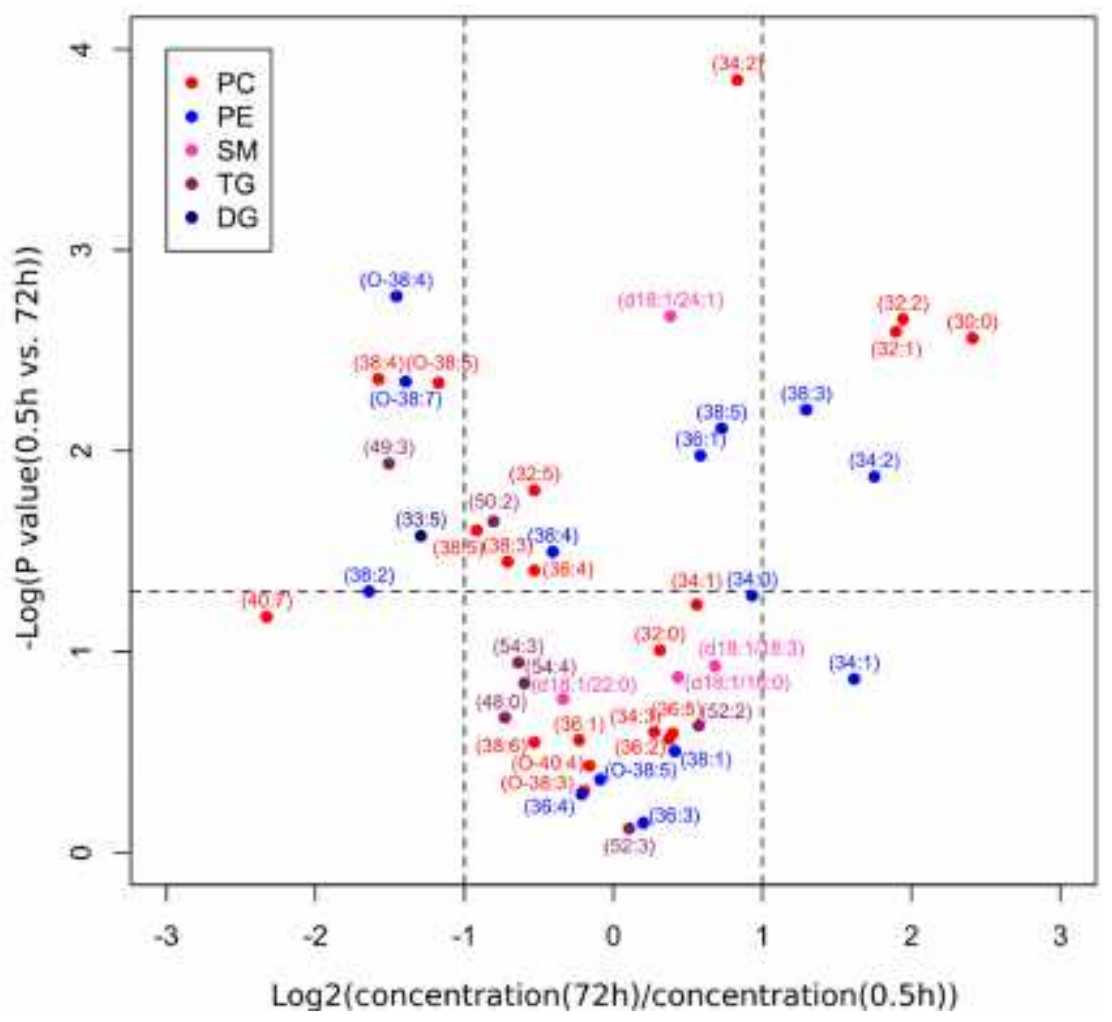

Figure 4. Volcano plot of lipid species concentration changes measured between 0.5 and 72 hours after activation. Fold-change indicates $\log 2$-transformed ratio of concentrations measured from activated cells at 0.5 and 72 hours. P-values were calculated using paired Student's t-test using data from samples cultured both in presence and absence of IL-4, comparing the $0.5 \mathrm{~h}$ and $72 \mathrm{~h}$ time points.

downregulation of varying magnitude of plasmenyl PC and PE species.

\subsection{Transcriptional regulation of lipid metabolism}

Comprehensive transcriptomics datasets from the human T-cell differentiation model have been published(14). To evaluate the role of transcriptional regulation in the lipid-level alterations, we correlated transcriptomic measurements with the results of lipidomic analysis. The focus was on genes coding for enzymes with documented roles in lipid metabolism, listed using annotations from KEGG database (Table 3)(15), complemented with additions based on recent literature. The broad functional classifications for these genes were fatty acid synthases, desaturases, elongases, acyl-CoA synthetases, or phospholipid acyltransferases, and known upstream regulators of these.

Although enzymatic activity is not controlled solely by means of mRNA-level change, in the case of fatty acid synthetase, elongases, and desaturases, the regulation reportedly takes place primarily at the transcript level(16). In line with this, the results indicated that within each functional category, several enzymes were subject to both transcriptional induction and repression. For example, expression of acetyl-CoA carbolyxase $\alpha$ (ACACA), a key enzyme in palmitate synthesis, was clearly induced, although level of Fatty acid synthase (FASN) remained relatively unchanged. A particularly large fold change was observed for the SCD gene, coding for stearyl-coenzyme A desaturase, an enzyme responsible for conversion of palmitic acid to palmitoleic acid, and stearic acid to oleic acid (Figure 5) SCD is co-regulated with fatty acid desaturases FADS1 and FADS2, as well as elongase ELOVL6, all of which were moderately increased with Tcell activation. FADS1 and FADS2 function as delta-5 and delta-6 desaturases, respectively. ELOVL6 in turn catalyzes elongation of 16-carbon fatty acids to 18 -carbon, and is specific for the saturated palmitic acid substrate, resulting in generation of stearic acid, which can be subsequently desaturated by SCD. The upstream regulators of this gene module include the PPAR $\alpha / \operatorname{RXR} \alpha$ pathway, also upregulated in T-cells within the same time scale(17). Interestingly, in addition to ELOVL6, expression of ELOVL1, which is also specific for saturated fatty acids, appeared to be induced, despite not being directly coregulated by the PPAR $\alpha$ pathway.

Incorporation of both endogenously synthesized and dietary fatty acids to phospholipids takes place either through de novo synthesis via the Kennedy pathway(18, 19) or by means of remodeling via the Lands' cycle(20). The rate-limiting reaction of the Kennedy pathway is catalyzed by phosphocholine cytidylyltransferase (CT), the activity of which is regulated at least partly by phosphorylation(21). Nevertheless, a clear induction of 
Table 3. Transcriptomic changes ( $\log 2)$ in activated cells in comparison to unstimulated condition

\begin{tabular}{|c|c|c|c|c|c|c|c|c|c|c|}
\hline Gene/probe ID & KEGG category & $0.5 \mathrm{~h}$ & $1 \mathrm{~h}$ & $2 \mathrm{~h}$ & 4h & $6 h$ & $12 \mathrm{~h}$ & $24 \mathrm{~h}$ & $48 \mathrm{~h}$ & $72 \mathrm{~h}$ \\
\hline SCD 200832_s_at & Desaturase & -0.25 & 0.03 & 0.76 & 0.74 & 1.62 & 3.12 & 3.80 & 4.61 & 5.22 \\
\hline SCD 200831_s_at & Desaturase & -0.10 & -0.02 & -0.26 & 0.03 & 0.20 & 0.58 & 1.16 & 1.63 & 1.72 \\
\hline SCD 211708_s_at & Desaturase & 0.20 & 0.00 & 0.33 & 0.31 & 0.56 & 1.13 & 1.72 & 3.05 & 2.73 \\
\hline SCD 211162_x_at & Desaturase & -0.13 & 0.02 & -0.07 & -0.03 & 0.04 & 0.46 & 0.61 & 1.39 & 1.27 \\
\hline SCD5 220232_at & Desaturase & -0.05 & -0.01 & -0.18 & -0.07 & -0.22 & -0.54 & -0.41 & -0.47 & -0.46 \\
\hline SCD5 224901_at & Desaturase & 0.05 & -0.06 & 0.04 & -0.20 & 0.04 & 0.18 & 0.60 & 0.56 & 0.50 \\
\hline SCD5 224018_s_at & Desaturase & 0.13 & 0.10 & -0.04 & 0.16 & -0.03 & 0.08 & 0.08 & 0.30 & 0.33 \\
\hline FADS1 208962_s_at & Desaturase & -0.18 & -0.21 & -0.25 & -0.18 & 0.00 & 2.24 & 3.27 & 3.80 & 3.88 \\
\hline FADS1 208963_x_at & Desaturase & 0.14 & 0.20 & -0.24 & -0.06 & -0.25 & 1.08 & 1.89 & 2.38 & 2.53 \\
\hline FADS1 208964_s_at & Desaturase & -0.10 & -0.01 & 0.01 & 0.02 & 0.01 & 1.56 & 2.86 & 3.33 & 3.49 \\
\hline FADS2 202218_s_at & Desaturase & 0.12 & 0.13 & 0.11 & 0.31 & -0.13 & 0.58 & 0.84 & 1.39 & 1.40 \\
\hline FADS3 216080_s_at & Desaturase & -0.29 & -0.13 & -0.23 & -0.33 & -0.33 & -0.14 & -0.07 & -0.01 & -0.09 \\
\hline FADS3 204257_at & Desaturase & 0.12 & 0.06 & 0.25 & -0.06 & -0.20 & 0.00 & 0.24 & 0.20 & 0.11 \\
\hline DEGS2 236496_at & Desaturase & 0.09 & -0.06 & 0.08 & 0.06 & 0.27 & 0.02 & 0.15 & 0.12 & 0.15 \\
\hline ELOVL1 218028_at & Elongase & -0.29 & 0.15 & -0.02 & 0.01 & 0.57 & 1.18 & 1.14 & 1.28 & 1.21 \\
\hline ELOVL1 57163_at & Elongase & -0.20 & -0.20 & -0.03 & 0.30 & 0.62 & 1.25 & 1.21 & 1.40 & 1.31 \\
\hline ELOVL2 213712_at & Elongase & 0.09 & 0.09 & 0.01 & 0.08 & -0.07 & 0.09 & 0.02 & 0.09 & 0.13 \\
\hline ELOVL2 220029_at & Elongase & 0.07 & 0.11 & 0.13 & 0.09 & -0.12 & -0.01 & 0.01 & 0.08 & 0.19 \\
\hline ELOVL4 219532_at & Elongase & 0.20 & 0.36 & 0.27 & 0.23 & 0.29 & 0.31 & 0.34 & 0.40 & 0.53 \\
\hline ELOVL5 208788_at & Elongase & -0.06 & 0.00 & 0.18 & 0.23 & 0.50 & 0.75 & 0.65 & 0.71 & 1.40 \\
\hline ELOVL5 215082_at & Elongase & $\begin{array}{ll}-0.11 \\
\end{array}$ & -0.26 & -0.51 & -0.31 & -0.39 & -0.49 & -0.30 & 0.22 & 0.74 \\
\hline ELOVL6 204256_at & Elongase & 0.15 & 0.37 & 0.21 & 1.19 & 1.47 & 1.88 & 1.18 & 1.77 & 1.98 \\
\hline ELOVL6 210868_s_at & Elongase & 0.05 & 0.00 & -0.10 & 0.23 & 0.33 & 0.51 & 0.30 & 0.32 & 0.57 \\
\hline ELOVL6 227491_at & Elongase & -0.13 & $\begin{array}{c}-0.18 \\
\end{array}$ & -0.13 & -0.29 & 0.06 & 0.17 & -0.11 & -0.09 & 0.14 \\
\hline ELOVL7 227180_at & Elongase & -0.18 & -0.07 & 0.25 & 0.37 & 0.23 & -0.02 & 0.09 & 0.10 & 0.24 \\
\hline ELOVL3 234513_at & Elongase & 0.23 & 0.06 & 0.20 & 0.15 & 0.15 & 0.12 & 0.08 & 0.14 & 0.12 \\
\hline FASN 212218_s_at & Fatty Acid Synthase & -0.16 & -0.04 & -0.44 & -0.49 & -0.26 & 0.38 & 0.37 & 0.31 & -0.08 \\
\hline FASN 217006_x_at & Fatty Acid Synthase & 0.01 & -0.10 & -0.02 & 0.17 & 0.04 & -0.02 & 0.01 & -0.04 & -0.07 \\
\hline MCAT 213132_s_at & Fatty Acid Synthase & 0.31 & 0.81 & 1.02 & 1.53 & 1.98 & 1.84 & 1.79 & 1.31 & 0.13 \\
\hline HSD17B12 217869_at & Fatty Acid Synthase & -0.20 & -0.25 & 1.05 & 2.24 & 1.88 & 2.00 & 1.41 & 1.84 & 2.07 \\
\hline HSD17B12 1554122_a_at & Fatty Acid Synthase & 0.12 & 0.35 & 0.67 & 0.76 & 0.32 & -0.20 & -0.38 & 0.02 & -0.18 \\
\hline HSD17B12 1554121_at & Fatty Acid Synthase & -0.17 & -0.05 & 0.09 & -0.02 & 0.08 & -0.14 & -0.16 & -0.01 & 0.10 \\
\hline PTPLB 212640_at & Fatty Acid Synthase & -0.07 & -0.20 & -0.11 & 0.19 & 0.63 & 0.69 & 0.40 & -0.11 & -0.07 \\
\hline PTPLB 227741_at & Fatty Acid Synthase & -0.22 & -0.05 & 0.40 & 0.89 & 0.81 & 0.14 & -0.15 & -0.08 & -0.22 \\
\hline PTPLA 219654_at & Fatty Acid Synthase & -0.07 & -0.20 & -0.22 & -0.11 & -0.06 & 0.35 & 0.37 & 0.09 & 0.25 \\
\hline TECR 208336_s_at & Fatty Acid Synthase & -0.25 & -0.16 & -0.17 & -0.60 & -0.93 & -0.31 & 0.33 & 0.51 & 0.66 \\
\hline OLAH 219975_x_at & Fatty Acid Synthase & 0.14 & 0.13 & 0.08 & 0.07 & $\begin{array}{l}-0.01 \\
\end{array}$ & -0.08 & 0.03 & -0.07 & 0.53 \\
\hline OLAH 222945_x_at & Fatty Acid Synthase & 0.19 & 0.20 & 0.16 & -0.03 & -0.09 & 0.01 & 0.00 & 0.26 & 0.41 \\
\hline OLAH 233126_s_at & Fatty Acid Synthase & 0.06 & 0.04 & 0.11 & -0.04 & -0.05 & -0.10 & -0.14 & -0.01 & 0.31 \\
\hline PPT1 200975_at & Fatty Acid Synthase & -0.03 & -0.16 & -0.23 & 0.64 & 0.68 & 0.76 & 0.82 & 0.35 & 0.27 \\
\hline PPT2 209826_at & Fatty Acid Synthase & -0.35 & -0.22 & -0.44 & -0.71 & -0.56 & -0.62 & -0.98 & -0.71 & -0.63 \\
\hline PPT2 208469_s_at & Fatty Acid Synthase & -0.38 & -0.35 & -0.33 & -0.47 & -0.24 & -0.23 & -0.23 & -0.37 & -0.21 \\
\hline PPT2 209490_s_at & Fatty Acid Synthase & 0.03 & 0.04 & 0.29 & 0.09 & 0.18 & 0.29 & 0.18 & 0.08 & 0.09 \\
\hline ACOT7 208002_s_at & Fatty Acid Synthase & -0.32 & -0.23 & 0.22 & 0.36 & 1.18 & 3.31 & 4.33 & 3.87 & 3.07 \\
\hline ACOT7 215728_s_at & Fatty Acid Synthase & -0.07 & 0.08 & 0.01 & 0.02 & 0.23 & 0.93 & 1.35 & 1.21 & 0.70 \\
\hline ACOT4 229534_at & Fatty Acid Synthase & -0.07 & -0.11 & 0.33 & 0.50 & 0.28 & 1.11 & 0.97 & 0.43 & 0.33 \\
\hline ACOT1 202982_s_at & Fatty Acid Synthase & 0.27 & 0.77 & 0.37 & -0.72 & -0.73 & -0.71 & -0.51 & -0.59 & -0.45 \\
\hline GPAT2 235557_at & Phospholipid acyltransferase & -0.32 & -0.12 & -0.23 & 0.30 & 0.76 & 1.55 & 2.28 & 1.68 & 1.55 \\
\hline GPAM 225420_at & Phospholipid acyltransferase & -0.03 & -0.35 & -0.66 & 0.02 & 0.01 & -0.10 & -0.48 & -0.86 & -0.90 \\
\hline GPAM 225424_at & Phospholipid acyltransferase & 0.45 & 0.02 & -0.34 & 0.27 & 0.57 & 0.88 & 0.52 & -0.13 & -0.41 \\
\hline AGPAT6 229577_at & Phospholipid acyltransferase & 0.04 & 0.31 & -0.03 & -0.08 & -0.32 & -0.23 & -0.40 & -0.68 & -0.52 \\
\hline AGPAT6 224776_at & Phospholipid acyltransferase & -0.11 & 0.11 & -0.14 & -0.27 & -0.07 & -0.02 & -0.19 & -0.41 & -0.22 \\
\hline AGPAT9 224480_s_at & Phospholipid acyltransferase & 0.16 & 1.20 & 1.34 & -0.01 & 0.02 & -0.22 & -0.28 & -0.37 & -0.60 \\
\hline AGPAT1 215535_s_at & Phospholipid acyltransferase & -0.61 & -0.44 & -0.41 & -0.31 & -0.07 & 0.25 & 0.86 & 0.56 & 0.64 \\
\hline AGPAT1 32836_at & Phospholipid acyltransferase & -0.38 & -0.33 & $\begin{array}{l}-0.39 \\
\end{array}$ & -0.31 & -0.38 & -0.03 & 0.42 & 0.31 & 0.39 \\
\hline AGPAT2 210678_s_at & Phospholipid acyltransferase & -0.17 & -0.27 & -0.12 & 0.12 & 0.02 & 0.34 & 0.84 & 0.48 & 0.86 \\
\hline AGPAT2 32837_at & Phospholipid acyltransferase & -0.06 & -0.07 & -0.19 & -0.05 & 0.02 & 0.23 & 0.68 & 0.59 & 0.69 \\
\hline AGPAT3 223183_at & Phospholipid acyltransferase & -0.02 & 0.13 & 1.26 & 1.30 & 1.20 & 1.48 & 1.54 & 0.81 & 0.99 \\
\hline AGPAT3 225440_at & Phospholipid acyltransferase & -0.54 & -0.62 & 0.07 & 0.75 & 0.40 & 0.87 & 1.31 & 1.08 & 1.12 \\
\hline AGPAT3 223184_s_at & Phospholipid acyltransferase & -0.20 & -0.05 & 0.67 & 0.54 & 0.20 & 0.50 & 0.62 & 0.43 & 0.77 \\
\hline AGPAT3 219723_x_at & Phospholipid acyltransferase & -0.37 & -0.28 & -0.19 & 0.00 & -0.05 & -0.09 & 0.03 & -0.21 & -0.04 \\
\hline AGPAT3 224282_s_at & Phospholipid acyltransferase & -0.45 & -0.10 & 0.01 & 0.09 & -0.14 & 0.23 & 0.65 & 0.39 & -0.01 \\
\hline AGPAT3 223182_s_at & Phospholipid acyltransferase & -0.09 & -0.17 & 0.93 & 0.96 & 0.68 & 0.51 & 0.40 & 0.27 & 0.64 \\
\hline ABHD5 218739_at & Phospholipid acyltransferase & 0.25 & 0.29 & 0.13 & 0.14 & 0.42 & 0.54 & 0.95 & 0.64 & 0.43 \\
\hline LCLAT1 226996_at & Phospholipid acyltransferase & -0.19 & -0.62 & 0.44 & 0.98 & 1.01 & 0.92 & 0.97 & 1.02 & 0.76 \\
\hline TAZ 203977_at & Phospholipid acyltransferase & -0.31 & -0.55 & -0.80 & -1.17 & -0.72 & -0.30 & -0.15 & -0.06 & 0.11 \\
\hline TAZ 37278_at & Phospholipid acyltransferase & -0.23 & -0.31 & -0.66 & -0.86 & -0.61 & -0.41 & -0.40 & -0.14 & 0.00 \\
\hline MBOAT2 226726_at & Phospholipid acyltransferase & 0.00 & -0.17 & -0.15 & -0.31 & -0.33 & -0.12 & 0.42 & 0.39 & 0.62 \\
\hline MBOAT2 213288_at & Phospholipid acyltransferase & 0.23 & 0.23 & -0.14 & -0.12 & -0.15 & -0.05 & 0.04 & -0.21 & -0.07 \\
\hline LPCAT1 201818_at & Phospholipid acyltransferase & -0.35 & -0.36 & -0.16 & 0.61 & 1.22 & 1.96 & 1.62 & 0.82 & 0.75 \\
\hline LPCAT2 222833_at & Phospholipid acyltransferase & 0.11 & 0.26 & 0.19 & 0.36 & 0.15 & 0.22 & 0.25 & 0.36 & 0.35 \\
\hline LPCAT2 227889_at & Phospholipid acyltransferase & -0.14 & 0.03 & 0.12 & 0.87 & 0.25 & 0.42 & 0.91 & 0.30 & 0.38 \\
\hline LPCAT2 239598_s_at & Phospholipid acyltransferase & 0.02 & 0.21 & 0.28 & 0.20 & -0.12 & -0.06 & 0.17 & 0.15 & 0.25 \\
\hline
\end{tabular}




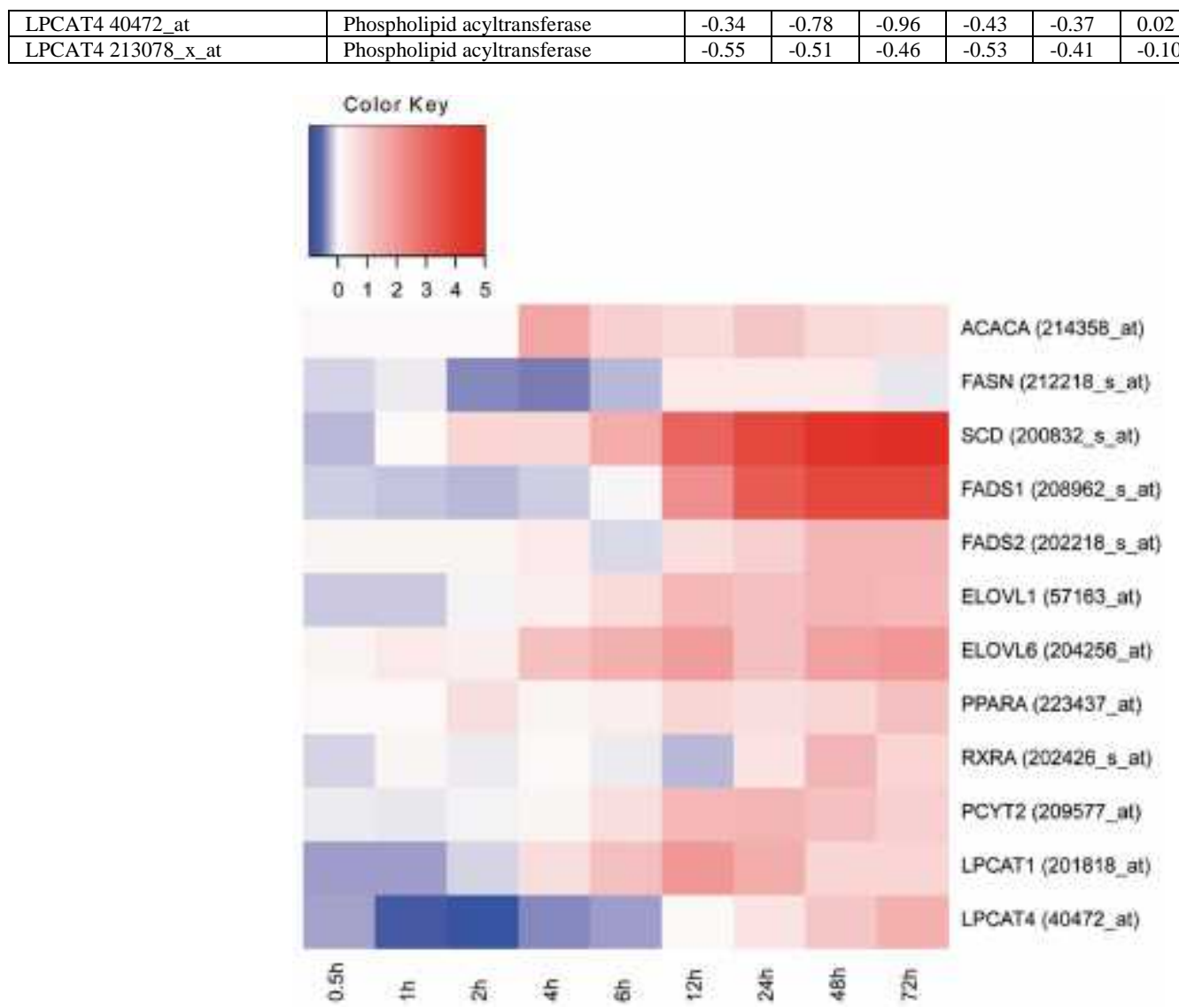

Figure 5. T-cell receptor activation associated transcriptional upregulation of lipid biosynthetic genes. Values represent log2 fold changes of CD3/CD28-activated cells compared to unstimulated cells.

PCYT2 gene, coding for phosphoethanolamine cytidylyltransferase was detected. In the Lands' cycle, glycerophospholipid fatty acid chains are remodeled by successive activities of phospholipase $\mathrm{A}_{2} \mathrm{~s}\left(\mathrm{PLA}_{2}\right)$ and enzymes of the lysophospholipid acyltransferase (LPLAT) family, the latter of which exhibit clear preferences regarding both acceptor phopsholipid head group and substrate side chain structure. Among the LPLAT probes in the microarray data, the ones for LPCAT1 and LPCAT4 indicated subtle upregulation towards the latest time points. LPCAT1 was first characterized as an acyltransferase responsible for generation of surfactant dipalmitoyl PC by alveolar cells, possessing specificity for 16:0 acyl-CoA, while LPCAT4 has recently been described as an 18:1specific acyltransferase(22-24). Altogether, the transcriptomics data supports the observation that the T-cell lipidome is actively and specifically regulated in TCRstimulated cells during development of functionally mature effector subsets.

\section{DISCUSSION}

While $\mathrm{CD}^{+}$T-cells and their activation and differentiation have been studied relatively thoroughly at the level of transcriptomics, proteomics, and most recently by epigenetic approaches, previous lipid-level investigations have been limited to experiments targeting specific molecular species and properties of local sub cellular systems, such as the T-cell receptor environment. So far, the development of activated effector T-cells has not been studied extensively at the level of molecular lipids. In the present study, we used a UPLC-MS-based lipidomic strategy in the analysis of human umbilical cord blood $\mathrm{CD} 4^{+} \mathrm{T}$-cells in both the unstimulated naïve state, and during a 72-hour time course following $\mathrm{T}$-cell receptor mediated activation, and IL-4-induced Th2 differentiation. Cord blood CD4 ${ }^{+}$ cells represent one of the most naïve obtainable population of human $\mathrm{T}$ helper cells, providing an attractive model system for studying early activation and polarization of human T-cells.

From the unstimulated $\mathrm{T}$ helper precursor (Thp) cells, we have catalogued the concentrations of 41 cellular lipids, representing the most common glycerophospholipids, sphingomyelins, and triglycerides. To our knowledge this represents the most thorough lipidomic characterization of human primary T-cells to date, even though detection of lower abundance species was prohibited by limited availability of primary cell material. Moreover, some significant lipid classes such as phosphatidylinositols (PI), phosphatidylserines (PS), and cholesterol were not included in the data due to methodological limitations. Neither of these analytes are 
directly compatible with positive electrospray ionization and their analysis by MS necessitates either derivatization or alternative ionization strategies (ESI+)(10). Other notable categories outside the scope of our study included glycolipids and cardiolipin.

The lipidomic analysis was repeated in quadruplicate using pooled primary cell samples from multiple individuals to take into account biological variability due to diet and other environmental factors. The extent of relative variation was highest in the case of triglycerides, while the concentrations of abundant PC species followed a more consistent pattern. Triglycerides provide the body's storage of fatty acids in the form of intracellular lipid droplets, and thus are arguably the primary subject to dietary variation. The lipidome of a related Jurkat T-cell line has been recently investigated by Zech et al.(25). Systematic comparison of these datasets indicated no fundamental differences in terms of phospholipid abundance distribution.

The majority of cellular phospholipids are localized in cell membranes, primarily in the plasma membrane, where their distribution is asymmetric - outer leaflet consisting mainly of sphingolipids, glycosphingolipids, and phosphatidylcholine while inner leaflet is formed by glycerophospholipids such as phosphatidylserine and phosphatidylethanolamine(26-31). This organization is actively maintained by phospholipid flippase enzymes(32). Moreover, plasma membrane lipids are laterelly distributed in cholesterol- and glycosphingolipid-enriched-microdomains or "lipid rafts", providing distinct lipid surroundings for transmembrane protein complexes(33, 34). In T-cells, these lipid microdomains play an important role in T-cell receptor mediated activation, bringing together LAT, LCK, and stimulatory receptors while isolating inhibitory molecules such as CD45(5). Conceptually, this microdomain organization has been proposed to facilitate switch-like signaling, where activation rate remains relatively constant even when antigen stimulation varies within a certain threshold(34). Although mechanisms of T-cell receptor clustering have been shown to involve regulation by protein interactions, direct lipidomic analyses of T-cell derived HIV particles as well as immunoisolated TCR raft fractions have verified that the TCR membrane fraction is physically distinct from plasma membrane and is relatively enriched in SM, cholesterol, and saturated $\mathrm{PCs}(35,36)$. Furthermore, using stimulated emission depletion (STED) fluorescence microscopy, lateral organization of sphingolipids and cholesterol has recently been successfully observed also in living cells(37).

Although the subcellular distribution of lipids was beyond the scope of this study, we were able to measure notable changes in levels of several glycerophospholipids following T-cell activation. In general, the relative concentration of longer polyunsaturated glycerophospholipids decreased towards the end of the measured time series, while shorter, more saturated, lipids species exhibited clear corresponding increase at 48 and 72 hours. This time scale correlates with first rounds of cell division after activation, suggesting that membrane properties of newly generated daughter cells differ from the nonstimulated progenitors. On the level of relative concentrations of individual lipid species, these changes were most significant in case of upregulation of PCs (30:0), (32:1), and (32:2), and PEs (38:3) and (34:2), and relative decrease of PCs (38:4) and (O-38:5), and PEs $(\mathrm{O}-38: 7)$ and (O-38:4). As mammalian fatty acid biosynthesis produces predominantly species with 14 to 18 carbons and a low degree of saturation(38), it might be suggested that the observed lipid concentration changes reflect upregulation of endogenous fatty acid biosynthesis and a relative decrease of dietary fatty acid in cellular membranes. Interestingly, in macrophages, PC synthesis has been demonstrated to be prerequisite for cytokine secretion(39).

Recent reports have demonstrated that expression of raft-associated lipids, such as ganglioside GM1, lactosylceramide, glucosylceramide, ceramide, sphingomyelin and cholesterol, is upregulated in response to activation through TCR and CD28(40-43). At least in the cases of GM1 and cholesterol, the upregulation is dependent on increased de novo synthesis $(41,43)$. In view of these observations the question arises whether the lipidomic changes observed in the present data also reflect upregulation of raft components. Specifically, saturated PC species with 1 or 0 double bonds and 34 or less fatty acyl carbons have been reported to be enriched in TCR rafts, while longer and less saturated species are depleted in relation to total lipidome(36). However, of the most significantly enriched PC species (34:1), (32:0), and (30:0), only (30:0) was clearly upregulated in our data. Moreover, the raft data by Zech et al. does not show any enrichment of saturated PE species in the TCR fractions. Overall, it was not apparent that association with TCR rafts would explain all the observed TCR-induced changes in primary T-cell lipidome.

The greatly increased metabolic demands of activated T-cells are highlighted by a shift of glucose catabolism from oxidative phosphorylation to glycolysis. This reversible transition to so called Warburg metabolism has been proposed as a way for a cell to maximize the accumulation of new biomass in conditions of non-limiting glucose concentration(44), and is correlated with upregulation of nutrient uptake in activated cells. This mechanism is associated with the highly elevated proliferation rate of activated T-cells, and is typically employed also by cancer cells. Recent evidence has shown that in breast cancer, the Warburg effect is accompanied by upregulation of de novo fatty acid synthesis, and subsequent alteration of plasma membrane properties(45). Among others, concentration of $\mathrm{PC}(14: 0 / 16: 0)$ was consistently increased in tumor cells, and was associated with tumor progression, resembling the observed relative increase of $\mathrm{PC}(30: 0)$ in the activated T-cells.

The transcriptomic regulation of activated T-cells in an identical experimental system has been previously investigated(14). Although regulatory pathways of lipid metabolism are complex and still incompletely known, 
integration of the mRNA and lipid datasets provided several potential regulatory mechanisms acting in response to T-cell activation. Most strikingly, expression of the stearoyl CoA-desaturase (SCD) gene was greatly upregulated in activated cells. SCD catalyzes n-9 desaturation of 16:0 and 18:0 fatty acids, and is a regulatory node for fatty acid metabolism, highlighted by the fact that SCD knockout mice have severely impaired lipid synthesis(46). Interestingly, in M-CSF-induced macrophage differentiation, SCD increases monounsaturated fatty acids and leads to reduced amount of polyunsaturated fatty acids(47). SCD is regulated by SREBP-1/2, LXR $\alpha$, $\mathrm{RXR} \alpha$, and PPAR $\alpha(48,49)$. Of these, both RXR $\alpha$ and PPAR $\alpha$ were found to be moderately upregulated in response to TCRactivation in human $\mathrm{CD}^{+}$T-cells, providing a possible regulatory pathway. In addition to SCD, the FADS1 and FADS2 desaturases were induced in response to T-cell activation, as well as elongase ELOVL6. ELOVL6, ELOVL3, and SCD have been shown to be part of a regulatory network that controls lipid homeostasis(16, 49). These emergent regulatory properties might also potentially underlie the specific concentration changes seen in activated T-cells. In addition to enzymes of fatty acid synthesis pathways, some changes in regulation of glycerophospholipid metabolism were detected. Namely, upregulation of Pcyt2 and specific lysophospholipid acetyltransferases suggest that T-cell lipid composition might be specifically regulated on multiple levels.

In the present data the lipid kinetics in cells stimulated in presence or absence of IL-4 were nearly identical, suggesting that TCR-activation delivers a substantially stronger signal in terms of metabolic change. This is in line with our previous transcriptome and proteome level investigations, in which profound changes were measured in response to T-cell activation while cytokine-induced T-cell subset differentiation involved a more defined response $(14,50$, 51). However, Miguel et al. have recently demonstrated heterogeneity in human primary $\mathrm{CD}^{+}$T-cell plasma membrane organization with subsequent implications on cellular thresholds to activation and apoptosis, suggesting Th2polarized cells retaining a higher level of membrane order in comparison with Th1-population, as assessed using fluorescent membrane probes(13). Our global approach did not, unfortunately, allow a quantitative validation of these results. Notably, the role of mTOR as a central regulator of T-cell metabolism is becoming increasingly appreciated. An integrator of multiple environmental cues, mTOR regulates cell growth both spatially and temporally $(52,53)$. Recent evidence has indicated that integration of environmental signals by mTOR is additionally involved in $\mathrm{T}$ helper cell subset differentiation(54). In the broader context, perturbation of nucleotide biosynthesis has been shown to skew Th1/Th2 balance, providing further evidence for distinct metabolic pathways in $\mathrm{T}$ helper cell subsets(55). The extent and precise role of metabolomic regulation during $\mathrm{T}$-cell subset differentiation therefore still requires further investigation.

Altogether, the present data illustrates remarkable plasticity in human T-cell lipid composition in response to activation by stimulation of $\mathrm{T}$ cell receptor and CD28 pathways. Transcriptomic data suggests that this plasticity is regulated at the RNA-level by activation of fatty acid biosynthetic pathways, resulting in increased incorporation of de novo produced fatty acids into the plasma membrane. While a decrease in phospholipid saturation is generally correlated with decreased membrane fluidity(56), whether the changes observed in this study have specific functional consequences on membrane function remains to be elucidated. Importantly, recent data from human adipocytes indicates that biophysical membrane properties can be actively maintained despite remodeling of membrane lipid components(57). In relation to specific cellular systems such as T-cells, important information on the spatial regulation of lipids could be achieved by parallel analysis of distinct sub cellular compartments. Each organelle confined by a lipid membrane bilayer, such as endoplasmic reticulum (ER), Golgi apparatus, and mitochondria, expresses a characteristic lipid composition, actively regulated by localized synthesis and lipid transport. Therefore, selective expansion or reduction of a specific cellular compartment could be underlying some of the lipidomic changes observed in this study. According to recent reports, amounts of both mitochondria and ER are regulated by macroautophagy during $\mathrm{T}$ cell development leading to significantly reduced mitochondrial and ER content in mature peripheral $\mathrm{T}$ cells $(58,59)$. In TCRactivated cells, macroautophagy is further induced, but targets primarily soluble cytosolic components, and correlates with a moderate increase in both mitochondrial number and size(60). Furthermore, human cells have been shown to contain distinct pools of cytoplasmic and nuclear phosphatidylcholine, the latter of which is predominantly saturated and potentially has a structural role in association with chromatin(61). Such targeted lipidomic approaches, however, will still require further development of suitable cellular fractionation methods(62). The results of this current study should provide a useful framework for such investigations.

\section{ACKNOWLEDGEMENTS}

Riitta Lahesmaa, and Matej Oresic contributed equally to this manuscript. We would like to thank all voluntary blood donors; the personnel of Turku University Hospital Department of Obstetrics and Gynaecology, Maternity Ward (Hospital District of Southwest Finland) for the sample collection. The work was supported by the Academy of Finland (Centre of Excellence in Molecular Systems Immunology and Physiology Research, 2012-2017, Decision No. 250114, and grants 207490 SYSBIO, 116639 , 115939, 140019), the European Commission Seventh Framework grants (EC-FP7-SYBILLA-201106, EC-FP7NANOMMUNE-214281 and EC-FP7-DIABIMMUNE202063), JDRF, The Sigrid Juselius Foundation, Turku University Hospital Grant, and Turku University Foundation. Marjo Hakkarainen is acknowledged for skillful technical assistance. Dr. Robert Moulder is acknowledged for revising the language of the manuscript.

\section{REFERENCES}

1. G. E. Kaiko, J. C. Horvat, K. W. Beagley and P. M. Hansbro: Immunological decision-making: how does the 
immune system decide to mount a helper T-cell response? Immunology, 123, 326-338 (2008)

2. J. Zhu and W. E. Paul: CD4 T cells: fates, functions, and faults. Blood, 112(5), 1557-69 (2008)

3. J. Zhu, H. Yamane and W. E. Paul: Differentiation of effector CD4 T cell populations. Annu Rev Immunol, 28, 44589 (2010)

4. O. Acuto, V. Di Bartolo and F. Michel: Tailoring T-cell receptor signals by proximal negative feedback mechanisms. Nat Rev Immunol, 8(9), 699-712 (2008)

5. J. E. Smith-Garvin, G. A. Koretzky and M. S. Jordan: T cell activation. Annu Rev Immunol, 27, 591-619 (2009)

6. P. A. van der Merwe and O. Dushek: Mechanisms for T cell receptor triggering. Nat Rev Immunol, 11, 47-55 (2011)

7. V. Mayya, D. H. Lundgren, S. I. Hwang, K. Rezaul, L. Wu, J. K. Eng, V. Rodionov and D. K. Han: Quantitative phosphoproteomic analysis of $\mathrm{T}$ cell receptor signaling reveals system-wide modulation of protein-protein interactions. Sci Signal, 2(84), ra46 (2009)

8. R. J. Lund, M. Loytomaki, T. Naumanen, C. Dixon, Z. Chen, H. Ahlfors, S. Tuomela, J. Tahvanainen, J. Scheinin, T. Henttinen, O. Rasool and R. Lahesmaa: Genome-wide identification of novel genes involved in early Th1 and Th2 cell differentiation. J Immunol, 178(6), 3648-60 (2007)

9. M. Oresic, V. A. Hanninen and A. Vidal-Puig: Lipidomics: a new window to biomedical frontiers. Trends in Biotechnology, 26(12), 647-652 (2008)

10. M. Pulfer and R. C. Murphy: Electrospray mass spectrometry of phospholipids. Mass Spectrom Rev, 22(5), 332-64 (2003)

11. M. R. Wenk: Lipidomics: new tools and applications. Cell, $143,888-895$ (2010)

12. Y. H. Huang and K. Sauer: Lipid signaling in T-cell development and function. Cold Spring Harb Perspect Biol, 2(11), a002428 (2010)

13. L. Miguel, D. M. Owen, C. Lim, C. Liebig, J. Evans, A. I. Magee and E. C. Jury: Primary human CD4+ T cells have diverse levels of membrane lipid order that correlate with their function. J Immunol, 186, 3505-3516 (2011)

14. L. L. Elo, H. Jarvenpaa, S. Tuomela, S. Raghav, H. Ahlfors, K. Laurila, B. Gupta, R. J. Lund, J. Tahvanainen, R. D. Hawkins, M. Oresic, H. Lahdesmaki, O. Rasool, K. V. Rao, T. Aittokallio and R. Lahesmaa: Genome-wide profiling of interleukin-4 and STAT6 transcription factor regulation of human Th2 cell programming. Immunity, 32(6), 852-62 (2010)

15. M. Kanehisa and S. Goto: KEGG: kyoto encyclopedia of genes and genomes. Nucleic Acids Res, 28(1), 27-30 (2000)
16. H. Guillou, D. Zadravec, P. G. Martin and A. Jacobsson: The key roles of elongases and desaturases in mammalian fatty acid metabolism: Insights from transgenic mice. Prog Lipid Res, 49(2), 186-99 (2010)

17. M. Miyazaki and J. M. Ntambi: Role of stearoylcoenzyme A desaturase in lipid metabolism. Prostaglandins Leukot Essent Fatty Acids, 68(2), 113-21 (2003)

18. E. P. Kennedy and S. B. Weiss: The function of cytidine coenzymes in the biosynthesis of phospholipides. J Biol Chem, 222(1), 193-214 (1956)

19. E. P. Kennedy: Biosynthesis of complex lipids. Fed Proc, 20, 934-40 (1961)

20. W. E. Lands: Metabolism of glycerolipides; a comparison of lecithin and triglyceride synthesis. J Biol Chem, 231(2), 883-8 (1958)

21. H. Sugimoto, C. Banchio and D. E. Vance: Transcriptional regulation of phosphatidylcholine biosynthesis. Prog Lipid Res, 47(3), 204-20 (2008)

22. H. Nakanishi, H. Shindou, D. Hishikawa, T. Harayama, R. Ogasawara, A. Suwabe, R. Taguchi and T. Shimizu: Cloning and characterization of mouse lungtype acyl-CoA:lysophosphatidylcholine acyltransferase 1 (LPCAT1). Expression in alveolar type II cells and possible involvement in surfactant production. $J$ Biol Chem, 281(29), 20140-7 (2006)

23. X. Chen, B. A. Hyatt, M. L. Mucenski, R. J. Mason and J. M. Shannon: Identification and characterization of a lysophosphatidylcholine acyltransferase in alveolar type II cells. Proc Natl Acad Sci U S A, 103(31), 117249 (2006)

24. D. Hishikawa, H. Shindou, S. Kobayashi, H. Nakanishi, R. Taguchi and T. Shimizu: Discovery of a lysophospholipid acyltransferase family essential for membrane asymmetry and diversity. Proc Natl Acad Sci U S A, 105(8), 2830-5 (2008)

25. T. Zech, C. S. Ejsing, K. Gaus, B. de Wet, A. Shevchenko, K. Simons and T. Harder: Accumulation of raft lipids in T-cell plasma membrane domains engaged in TCR signalling. EMBO J, 28(5), 466-76 (2009)

26. G. van Meer: Cellular lipidomics. The EMBO Journal, 24(18), 3159-3165 (2005)

27. A. Zachowski: Phospholipids in animal eukaryotic membranes: transverse asymmetry and movement. Biochem J, 294 ( Pt 1), 1-14 (1993)

28. X. Han and R. W. Gross: Shotgun lipidomics: electrospray ionization mass spectrometric analysis and quantitation of cellular lipidomes directly from crude extracts of biological samples. Mass Spectrom Rev, 24(3), 367-412 (2005) 
29. Y. Lange, M. H. Swaisgood, B. V. Ramos and T. L. Steck: Plasma membranes contain half the phospholipid and $90 \%$ of the cholesterol and sphingomyelin in cultured human fibroblasts. J Biol Chem, 264(7), 3786-93 (1989)

30. D. E. Warnock, C. Roberts, M. S. Lutz, W. A. Blackburn, W. W. Young, Jr. and J. U. Baenziger: Determination of plasma membrane lipid mass and composition in cultured Chinese hamster ovary cells using high gradient magnetic affinity chromatography. J Biol Chem, 268(14), 10145-53 (1993)

31. A. Shevchenko and K. Simons: Lipidomics: coming to grips with lipid diversity. Nat Rev Mol Cell Biol, 11(8), 593-8 (2010)

32. X. Tang, M. S. Halleck, R. A. Schlegel and P. Williamson: A subfamily of P-type ATPases with aminophospholipid transporting activity. Science, 272(5267), 1495-7 (1996)

33. T. Harder and K. R. Engelhardt: Membrane domains in lymphocytes - from lipid rafts to protein scaffolds. Traffic, 5(4), 265-75 (2004)

34. K. Choudhuri and M. L. Dustin: Signaling microdomains in T cells. FEBS Lett, 584(24), 4823-31 (2010)

35. B. Brugger, B. Glass, P. Haberkant, I. Leibrecht, F. T. Wieland and H. G. Krausslich: The HIV lipidome: a raft with an unusual composition. Proc Natl Acad Sci U S A, 103(8), 2641-6 (2006)

36. T. Zech, C. S. Ejsing, K. Gaus, B. de Wet, A. Shevchenko, K. Simons and T. Harder: Accumulation of raft lipids in T-cell plasma membrane domains engaged in TCR signalling. The EMBO Journal, 28(5), 466-476 (2009)

37. C. Eggeling, C. Ringemann, R. Medda, G. Schwarzmann, K. Sandhoff, S. Polyakova, V. N. Belov, B. Hein, C. von Middendorff, A. Schonle and S. W. Hell: Direct observation of the nanoscale dynamics of membrane lipids in a living cell. Nature, 457(7233), 1159-62 (2009)

38. S. Smith: The animal fatty acid synthase: one gene, one polypeptide, seven enzymes. FASEB $J$, 8(15), 1248-59 (1994)

39. Y. Tian, C. Pate, A. Andreolotti, L. Wang, E. Tuomanen, K. Boyd, E. Claro and S. Jackowski: Cytokine secretion requires phosphatidylcholine synthesis. J Cell Biol, 181(6) 945-957 (2008)

40. M. Martin, H. Schneider, A. Azouz and C. E. Rudd: Cytotoxic T lymphocyte antigen 4 and CD28 modulate cell surface raft expression in their regulation of $\mathrm{T}$ cell function. J Exp Med, 194(11), 1675-81 (2001)

41. L. Tuosto, I. Parolini, S. Schroder, M. Sargiacomo, A. Lanzavecchia and A. Viola: Organization of plasma membrane functional rafts upon $\mathrm{T}$ cell activation. Eur $J$ Immunol, 31(2), 345-9 (2001)

42. S. Tani-ichi, K. Maruyama, N. Kondo, M. Nagafuku, K. Kabayama, J. Inokuchi, Y. Shimada, Y. Ohno-Iwashita, H. Yagita, S. Kawano and A. Kosugi: Structure and function of lipid rafts in human activated T cells. Int Immunol, 17(6), 749-58 (2005)

43. S. J. Bensinger, M. N. Bradley, S. B. Joseph, N. Zelcer, E. M. Janssen, M. A. Hausner, R. Shih, J. S. Parks, P. A. Edwards, B. D. Jamieson and P. Tontonoz: LXR signaling couples sterol metabolism to proliferation in the acquired immune response. Cell, 134(1), 97-111 (2008)

44. M. G. Vander Heiden, L. C. Cantley and C. B. Thompson: Understanding the Warburg effect: the metabolic requirements of cell proliferation. Science, 324(5930), 1029-33 (2009)

45. M. Hilvo, C. Denkert, L. Lehtinen, B. Muller, S. Brockmoller, T. Seppanen-Laakso, J. Budczies, E. Bucher, L. Yetukuri, S. Castillo, E. Berg, H. Nygren, M. Sysi-Aho, J. L. Griffin, O. Fiehn, S. Loibl, C. Richter-Ehrenstein, C. Radke, T. Hyotylainen, O. Kallioniemi, K. Iljin and M. Oresic: Novel theranostic opportunities offered by characterization of altered membrane lipid metabolism in breast cancer progression. Cancer Res, 71(9), 3236-45 (2011)

46. C. M. Paton and J. M. Ntambi: Biochemical and physiological function of stearoyl-CoA desaturase. Am J Physiol Endocrinol Metab, 297(1), E28-37 (2009)

47. J. Ecker, G. Liebisch, M. Grandl and G. Schmitz: Lower SCD expression in dendritic cells compared to macrophages leads to membrane lipids with less monounsaturated fatty acids. Immunobiology, 215(9-10), 748-55 (2010)

48. J. Ecker, G. Liebisch, M. Englmaier, M. Grandl, H. Robenek and G. Schmitz: Induction of fatty acid synthesis is a key requirement for phagocytic differentiation of human monocytes. Proc Natl Acad Sci U S A, 107(17), 7817-22 (2010)

49. C. D. Green, C. G. Ozguden-Akkoc, Y. Wang, D. B. Jump and L. K. Olson: Role of fatty acid elongases in determination of de novo synthesized monounsaturated fatty acid species. J Lipid Res, 51(7), 1871-7 (2010)

50. R. J. Lund, E. K. Ylikoski, T. Aittokallio, O. Nevalainen and R. Lahesmaa: Kinetics and STAT4- or STAT6-mediated regulation of genes involved in lymphocyte polarization to Th1 and Th2 cells. European Journal of Immunology, 33(4), 1105-1116 (2003)

51. R. Moulder, T. Lonnberg, L. L. Elo, J. J. Filen, E. Rainio, G. Corthals, M. Oresic, T. A. Nyman, T. Aittokallio and R. Lahesmaa: Quantitative proteomics analysis of the nuclear fraction of human CD4+ cells in the early phases of 
IL-4-induced Th2 differentiation. Mol Cell Proteomics, 9(9), 1937-53 (2010)

52. J. D. Powell and G. M. Delgoffe: The mammalian target of rapamycin: linking $\mathrm{T}$ cell differentiation, function, and metabolism. Immunity, 33(3), 301-11 (2010)

53. S. Wullschleger, R. Loewith and M. N. Hall: TOR signaling in growth and metabolism. Cell, 124(3), 471-84 (2006)

54. K. Lee, P. Gudapati, S. Dragovic, C. Spencer, S. Joyce, N. Killeen, M. A. Magnuson and M. Boothby: Mammalian target of rapamycin protein complex 2 regulates differentiation of Th1 and Th2 cell subsets via distinct signaling pathways. Immunity, 32(6), 743-53 (2010)

55. P. Dimitrova, A. Skapenko, M. L. Herrmann, R. Schleyerbach, J. R. Kalden and H. Schulze-Koops: Restriction of de novo pyrimidine biosynthesis inhibits Th1 cell activation and promotes Th2 cell differentiation. $J$ Immunol, 169(6), 3392-9 (2002)

56. C. D. Stubbs and A. D. Smith: The modification of mammalian membrane polyunsaturated fatty acid composition in relation to membrane fluidity and function. Biochim Biophys Acta, 779(1), 89-137 (1984)

57. K. H. Pietilainen, T. Rog, T. Seppanen-Laakso, S. Virtue, P. Gopalacharyulu, J. Tang, S. Rodriguez-Cuenca, A. Maciejewski, J. Naukkarinen, A. L. Ruskeepaa, P. S. Niemela, L. Yetukuri, C. Y. Tan, V. Velagapudi, S. Castillo, H. Nygren, T. Hyotylainen, A. Rissanen, J. Kaprio, H. YkiJarvinen, I. Vattulainen, A. Vidal-Puig and M. Oresic: Association of lipidome remodeling in the adipocyte membrane with acquired obesity in humans. PLoS Biol, 9(6), e1000623 (2011)

58. H. H. Pua, J. Guo, M. Komatsu and Y. W. He: Autophagy is essential for mitochondrial clearance in mature T lymphocytes. J Immunol, 182(7), 4046-55 (2009)

59. W. Jia, H. H. Pua, Q. J. Li and Y. W. He: Autophagy regulates endoplasmic reticulum homeostasis and calcium mobilization in T lymphocytes. J Immunol, 186(3), 156474 (2011)

60. V. M. Hubbard, R. Valdor, B. Patel, R. Singh, A. M. Cuervo and F. Macian: Macroautophagy regulates energy metabolism during effector $\mathrm{T}$ cell activation. J Immunol, 185(12), 7349-57 (2010)

61. A. N. Hunt, G. T. Clark, G. S. Attard and A. D. Postle: Highly saturated endonuclear phosphatidylcholine is synthesized in situ and colocated with CDP-choline pathway enzymes. J Biol Chem, 276(11), 8492-9 (2001)

62. G. van Meer: Cellular lipidomics. EMBO J, 24(18), 3159-65 (2005)

63. K. J. Rautajoki, E. M. Marttila, T. A. Nyman and R. Lahesmaa: Interleukin-4 inhibits caspase- 3 by regulating several proteins in the Fas pathway during initial stages of human $\mathrm{T}$ helper 2 cell differentiation. Molecular \& Cellular Proteomics, 6(2), 238-251 (2007)

64. W. de Koning and K. van Dam: A method for the determination of changes of glycolytic metabolites in yeast on a subsecond time scale using extraction at neutral $\mathrm{pH}$. Anal Biochem, 204(1), 118-23 (1992)

65. K. H. Pietilainen, M. Sysi-Aho, A. Rissanen, T. Seppanen-Laakso, H. Yki-Jarvinen, J. Kaprio and M. Oresic: Acquired Obesity Is Associated with Changes in the Serum Lipidomic Profile Independent of Genetic Effects - A Monozygotic Twin Study. PLoS ONE, 2(2), e218 (2007)

66. T. Pluskal, S. Castillo, A. Villar-Briones and M. Oresic: MZmine 2: Modular framework for processing, visualizing, and analyzing mass spectrometry-based molecular profile data. Bmc Bioinformatics, 11 (2010)

67. R. Laaksonen, M. Katajamaa, H. Paiva, M. Sysi-Aho, L. Saarinen, P. Junni, D. Lutjohann, J. Smet, R. Van Coster, T. Seppanen-Laakso, T. Lehtimaki, J. Soini and M. Oresic: A systems biology strategy reveals biological pathways and plasma biomarker candidates for potentially toxic statininduced changes in muscle. PLoS One, 1(1), e97 (2006)

Key Words: Lipid metabolism, Metabolomics, T-cell receptor activation, $\mathrm{T}$ helper cell differentiation

Send correspondence to: Riitta Lahesmaa, Tel: 35823338601 Fax: 35823338000, E-mail: rlahesma@btk.fi 